\title{
LOCAL CALIBRATIONS FOR MINIMIZERS OF THE MUMFORD-SHAH FUNCTIONAL WITH A REGULAR DISCONTINUITY SET
}

\section{Maria Giovanna MORA, Massimiliano MORINI}

S.I.S.S.A., via Beirut 2-4, 34014 Trieste, Italy

Received 3 October 2000, revised 16 January 2001

ABSTRACT. - Using a calibration method, we prove that, if $w$ is a function which satisfies all Euler conditions for the Mumford-Shah functional on a two-dimensional open set $\Omega$, and the discontinuity set $S_{w}$ of $w$ is a regular curve connecting two boundary points, then there exists a uniform neighbourhood $U$ of $S_{w}$ such that $w$ is a minimizer of the Mumford-Shah functional on $U$ with respect to its own boundary conditions on $\partial U$. We show that Euler conditions do not guarantee in general the minimality of $w$ in the class of functions with the same boundary value of $w$ on $\partial \Omega$ and whose extended graph is contained in a neighbourhood of the extended graph of $w$, and we give a sufficient condition in terms of the geometrical properties of $\Omega$ and $S_{w}$ under which this kind of minimality holds.

(C) 2001 L'Association Publications de l'Institut Henri Poincaré. Published by Elsevier B.V. All rights reserved

Keywords: Free-discontinuity problems; Calibration method

\section{Introduction}

This paper deals with local minimizers of the Mumford-Shah functional (see [8] and [9])

$$
\int_{\Omega}|\nabla u(x, y)|^{2} \mathrm{~d} x \mathrm{~d} y+\mathcal{H}^{1}\left(S_{u}\right),
$$

where $\Omega$ is a bounded open subset of $\mathbb{R}^{2}$ with a Lipschitz boundary, $\mathcal{H}^{1}$ is the onedimensional Hausdorff measure, $u$ is the unknown function in the space $S B V(\Omega)$ of special functions of bounded variation in $\Omega, S_{u}$ is the set of essential discontinuity points of $u$, while $\nabla u$ denotes its approximate gradient (see [2] or [3]).

Definition 1.1. - We say (as in [1]) that $u$ is a Dirichlet minimizer of (1.1) in $\Omega$ if it belongs to $\operatorname{SBV}(\Omega)$ and satisfies the inequality

$$
\int_{\Omega}|\nabla u(x, y)|^{2} \mathrm{~d} x \mathrm{~d} y+\mathcal{H}^{1}\left(S_{u}\right) \leqslant \int_{\Omega}|\nabla v(x, y)|^{2} \mathrm{~d} x \mathrm{~d} y+\mathcal{H}^{1}\left(S_{v}\right)
$$

E-mail addresses: mora@sissa.it (M.G. Mora), morini@sissa.it (M. Morini). 
for every function $v \in S B V(\Omega)$ with the same trace as $u$ on $\partial \Omega$.

Suppose that $u$ is a Dirichlet minimizer of (1.1) in $\Omega$ and that $S_{u}$ is a regular curve. Then the following equilibrium conditions are satisfied (see [8] and [9]):

(i) $u$ is harmonic on $\Omega \backslash S_{u}$;

(ii) the normal derivative of $u$ vanishes on both sides of $S_{u}$;

(iii) the curvature of $S_{u}$ is equal to the difference of the squares of the tangential derivatives of $u$ on both sides of $S_{u}$.

Elementary examples show that conditions (i), (ii), and (iii) are not sufficient for the Dirichlet minimality of $u$.

In this paper we prove that, if $S_{u}$ is an analytic curve connecting two points of $\partial \Omega$, then (i), (ii), (iii) are also sufficient for the Dirichlet minimality of $u$ in small domains. In other words, for every $\left(x_{0}, y_{0}\right)$ in $\Omega$, there is an open neighbourhood $U$ of $\left(x_{0}, y_{0}\right)$ such that $u$ is a Dirichlet minimizer of (1.1) in $U$. If $\left(x_{0}, y_{0}\right)$ does not lie on $S_{u}$, this fact is well known and can be proved by the calibration method (see [1]); so the interesting case is when we consider points belonging to $S_{u}$ : in this situation we have a stronger result, since we can prove that the Dirichlet minimality actually holds in a uniform neighbourhood of the discontinuity set. The analyticity assumption for $S_{u}$ does not seem too restrictive: it has been proved that the regular part of the discontinuity set of a minimizer is of class $C^{\infty}$ and it is a conjecture that it is analytic (see [3]).

Let us give the precise statement of the result.

THEOREM 1.2. - Let $\Omega_{0}$ be a connected open subset of $\mathbb{R}^{2}$ and $\Gamma$ be a simple analytic curve in $\Omega_{0}$ connecting two points of the boundary. Let $u$ be a function in $H^{1}\left(\Omega_{0} \backslash \Gamma\right)$ with $S_{u}=\Gamma$, with different traces at every point of $\Gamma$, and satisfying the Euler conditions (i), (ii), and (iii) in $\Omega_{0}$ (for the precise formulation of these conditions, see Section 2). Finally, let $\Omega$ be an open set with Lipschitz boundary, compactly contained in $\Omega_{0}$, such that $\Omega \cap \Gamma \neq \emptyset$. Then there exists an open neighbourhood $U$ of $\Gamma \cap \bar{\Omega}$ contained in $\Omega_{0}$ such that $u$ is a Dirichlet minimizer in $U$ of the Mumford-Shah functional (1.1).

This theorem generalizes the result of Theorem 4.2 of [5] in two directions: the discontinuity set $S_{u}$ can be any analytic curve and the Dirichlet minimality of $u$ is proved in a uniform neighbourhood of $S_{u} \cap \bar{\Omega}$. The proof is obtained, as in [5], by the calibration method introduced in [1]. The original idea of the new construction essentially relies on the definition of the calibration around the graph of $u$ : here it is obtained using the gradient field of a family of harmonic functions, whose graphs fibrate a neighbourhood of the graph of $u$. This technique seems to have some similarities with the classical method of the Weierstrass fields, where the proof of the minimality of a candidate $u$ is obtained by the construction of a slope field starting from a family of solutions of the Euler equation, whose graphs foliate a neighbourhood of the graph of $u$.

In this paper we are also interested in a different type of minimality: in Theorem 1.2 we compare $u$ with perturbations which can be very large, but concentrated in a fixed small domain; we wonder if a minimality property is preserved also when we admit as competitors perturbations of $u$ with $L^{\infty}$-norm very small outside a small neighbourhood of $S_{u}$, but support possibly coinciding with $\bar{\Omega}$.

This is made precise by the following definition. 
DEFINITION 1.3. - A function $u \in S B V(\Omega)$ is a local graph-minimizer in $\Omega$ if there exists a suitable neighbourhood $U$ of the extended graph $\Gamma_{u}$ of $u$ (for the notion of extended graph, see Section 2) such that

$$
\int_{\Omega}|\nabla u(x, y)|^{2} \mathrm{~d} x \mathrm{~d} y+\mathcal{H}^{1}\left(S_{u}\right) \leqslant \int_{\Omega}|\nabla v(x, y)|^{2} \mathrm{~d} x \mathrm{~d} y+\mathcal{H}^{1}\left(S_{v}\right)
$$

for every $v \in S B V(\Omega)$ with the same trace as $u$ on $\partial \Omega$ and whose extended graph $\Gamma_{v}$ is contained in $U$.

In [1] it is proved that any harmonic function defined on $\Omega$ is a local graph-minimizer whatever $\Omega$ is. If the function presents some discontinuities, what we discover is that the graph-minimality may fail when $\Omega$ is too large, even in the case of rectilinear discontinuities, as the counterexample given in Section 4 shows.

To get the graph-minimality we have to add some restrictions on the domain $\Omega$. To this aim we introduce a suitable quantity which seems useful to describe the right geometrical interaction between $S_{u}$ and $\Omega$. Given an open set $A$ (with Lipschitz boundary) and a portion $\Gamma$ of $\partial A$ (with nonempty relative interior in $\partial A$ ), we define $K(\Gamma, A)$ by the variational problem

$$
\begin{gathered}
K(\Gamma, A):=\inf \left\{\int_{A}|\nabla v(x, y)|^{2} \mathrm{~d} x \mathrm{~d} y: \quad v \in H^{1}(A), \int_{\Gamma} v^{2} \mathrm{~d} \mathcal{H}^{1}=1,\right. \\
\text { and } v=0 \text { on } \partial A \backslash \Gamma\} .
\end{gathered}
$$

First of all, it is easy to see that in the problem above the infimum is attained; moreover, the notation is well chosen since $K(\Gamma, A)$ is a quantity depending only on $\Gamma$ and $A$, which describes a kind of "capacity" of the prescribed portion of the boundary with respect to the whole open set. Note also that if $A_{1} \subset A_{2}$, and $\Gamma_{1} \subset \Gamma_{2}$, then $K\left(\Gamma_{1}, A_{1}\right) \geqslant K\left(\Gamma_{2}, A_{2}\right)$, which suggests that if $K(\Gamma, A)$ is very large, then $A$ is thin in some sense. It is convenient to give the following definition.

DEFINITION 1.4. - Given a simple analytic curve $\Gamma$, we say that an open set $\Omega$ is $\Gamma$-admissible if it is bounded, $\Gamma \cap \bar{\Omega}$ connects two points of $\partial \Omega$, and $\Omega \backslash \Gamma$ has two connected components, which have Lipschitz boundary.

The following theorem gives a sufficient condition for the graph-minimality in terms of $K(\Gamma, \Omega)$ and of the geometrical properties of the curve. We denote the length of $\Gamma$ by $l(\Gamma)$, its curvature by curv $\Gamma$, and the $L^{\infty}$-norm of curv $\Gamma$ by $k(\Gamma)$.

THEOREM 1.5. - Let $\Omega_{0}, \Omega, u$, and $\Gamma=S_{u}$ satisfy the same assumptions as in Theorem 1.2 ; suppose that $\Omega$ is $\Gamma$-admissible and denote by $\Omega_{1}$ and $\Omega_{2}$ the two connected components of $\Omega \backslash \Gamma$, by $u_{i}$ the restriction of $u$ to $\Omega_{i}$, and by $\partial_{\tau} u_{i}$ its tangential derivative on $\Gamma$. There exists an absolute constant $c>0$ (independent of $\Omega_{0}, \Omega, \Gamma$, 
and $u$ ) such that if

$$
\frac{\min _{i=1,2} K\left(\Gamma \cap \Omega, \Omega_{i}\right)}{1+l^{2}(\Gamma \cap \Omega)+l^{2}(\Gamma \cap \Omega) k^{2}(\Gamma \cap \Omega)}>c \sum_{i=1}^{2}\left\|\partial_{\tau} u_{i}\right\|_{C^{1}(\Gamma \cap \Omega)}^{2},
$$

then $u$ is a local graph-minimizer on $\Omega$.

Remark that condition (1.3) imposes a restriction on the size of $\Omega$ depending on the behaviour of $u$ along $S_{u}$ : if $u$ has large or very oscillating tangential derivatives, we have to take $\Omega$ quite small to guarantee that (1.3) is satisfied. In the special case of a locally constant function $u$, condition (1.3) is always fulfilled whatever the domain is; so $u$ is a local graph-minimizer whatever $\Omega$ is, in agreement with a result that will be proved in the final version of [1].

The plan of the paper is the following: in Section 2, we fix some notation and recall the main result of [1]; Section 3 contains the proof of Theorem 1.2; finally, Section 4 is devoted to the graph-minimality: we give a counterexample when (1.3) is violated, we prove Theorem 1.5, and present some qualitative properties of $K(\Gamma, \Omega)$.

\section{Preliminary results}

Given any subset $A$ of $\mathbb{R}^{2}$ and $\delta>0$, we denote by $A_{\delta}$ the $\delta$-neighbourhood of $A$, defined by

$$
A_{\delta}:=\left\{\left(x_{0}, y_{0}\right) \in \mathbb{R}^{2}: \exists(x, y) \in A \text { such that }\left|\left(x-x_{0}, y-y_{0}\right)\right|<\delta\right\} .
$$

Let $\Omega$ be an open set in $\mathbb{R}^{2}$. If $v \in S B V(\Omega)$, for every $\left(x_{0}, y_{0}\right) \in \Omega$ we put

$$
v^{+}\left(x_{0}, y_{0}\right):=\underset{(x, y) \rightarrow\left(x_{0}, y_{0}\right)}{\operatorname{ap} \limsup } v(x, y) \quad \text { and } \quad v^{-}\left(x_{0}, y_{0}\right):=\underset{(x, y) \rightarrow\left(x_{0}, y_{0}\right)}{\operatorname{ap} \liminf } v(x, y)
$$

(see [3]). We recall that $v^{+}=v^{-} \mathcal{H}^{1}$-a.e. in $\Omega \backslash S_{v}$, while for $\mathcal{H}^{1}$-a.e. $\left(x_{0}, y_{0}\right) \in S_{v}$

$$
v^{ \pm}\left(x_{0}, y_{0}\right)=\lim _{r \rightarrow 0^{+}} \frac{1}{\mathcal{L}^{2}\left(B_{r}^{ \pm}\left(x_{0}, y_{0}\right)\right)} \int_{B_{r}^{ \pm}\left(x_{0}, y_{0}\right)} v(x, y) \mathrm{d} x \mathrm{~d} y,
$$

where $B_{r}^{ \pm}\left(x_{0}, y_{0}\right)$ is the intersection of the ball of radius $r$ centred at $\left(x_{0}, y_{0}\right)$ with the half-space $\left\{(x, y) \in \mathbb{R}^{2}: \pm\left(x-x_{0}, y-y_{0}\right) \cdot v_{v}\left(x_{0}, y_{0}\right) \geqslant 0\right\}$, where the vector $v_{v}\left(x_{0}, y_{0}\right)$ is the normal vector to $S_{v}$ at $\left(x_{0}, y_{0}\right)$ (which is defined $\mathcal{H}^{1}$-a.e. on $\left.S_{v}\right)$. The extended graph of $v$ is the set

$$
\Gamma_{v}:=\left\{(x, y, t) \in \Omega \times \mathbb{R}: v^{-}(x, y) \leqslant t \leqslant v^{+}(x, y)\right\} .
$$

Let $\Gamma$ be a smooth curve in $\Omega$. Fix an orientation of $\Gamma$ and call $v$ the corresponding normal vector field to $\Gamma$. Let $\xi \mapsto(x(\xi), y(\xi))$ be a parameterization of $\Gamma$ by the arclength. The (signed) curvature is defined by

$$
\operatorname{curv} \Gamma(\xi)=-(\ddot{x}(\xi), \ddot{y}(\xi)) \cdot v(\xi) ;
$$


since the two vectors in (2.1) are parallel, it follows that

$$
[\operatorname{curv} \Gamma(\xi)]^{2}=(\ddot{x}(\xi))^{2}+(\ddot{y}(\xi))^{2}
$$

Let $u \in \operatorname{SBV}(\Omega)$ be a function such that $S_{u}=\Gamma$. We say that $u$ satisfies the Euler conditions for the Mumford-Shah functional in $\Omega$ if

(i) $u$ is harmonic in $\Omega \backslash \Gamma$ and $u \in H^{1}(\Omega \backslash \Gamma)$,

(ii) $\partial u / \partial v=0$ on $\Gamma$,

(iii) $\left|\nabla u^{+}\right|^{2}-\left|\nabla u^{-}\right|^{2}=\operatorname{curv} \Gamma$ at every point of $\Gamma$, where $\nabla u^{ \pm}$denote the traces of $\nabla u$ on $\Gamma$.

If $U$ is any open subset of $\mathbb{R}^{3}$, we shall consider the collection $\mathcal{F}(U)$ of all piecewise $C^{1}$ vector fields $\varphi: U \rightarrow \mathbb{R}^{2} \times \mathbb{R}$ with the following property: there exists a finite family $\left(A_{i}\right)_{i \in I}$ of pairwise disjoint open subsets of $U$ such that the family of their closures covers $U, \partial A_{i} \cap U$ is a Lipschitz surface without boundary for every $i \in I$, and $\left.\varphi\right|_{A_{i}} \in C^{1}\left(\overline{A_{i}}, \mathbb{R}^{2} \times \mathbb{R}\right)$.

For every vector field $\varphi: U \rightarrow \mathbb{R}^{2} \times \mathbb{R}$ we define the maps $\varphi^{x}, \varphi^{y}, \varphi^{z}: U \rightarrow \mathbb{R}$ by

$$
\varphi(x, y, z)=\left(\varphi^{x}(x, y, z), \varphi^{y}(x, y, z), \varphi^{z}(x, y, z)\right) .
$$

Let $U$ be an open neighbourhood of $\Gamma_{u}$ such that the intersection with every straight vertical line is connected. A calibration for $u$ in $U$ is a bounded vector field $\varphi \in \mathcal{F}(U)$ which is continuous on the graph of $u$ and satisfies the following properties:

(a) $\operatorname{div} \varphi=0$ in the sense of distributions in $U$;

(b) $\left(\varphi^{x}(x, y, z)\right)^{2}+\left(\varphi^{y}(x, y, z)\right)^{2} \leqslant 4 \varphi^{z}(x, y, z)$ at every continuity point $(x, y, z)$ of $\varphi$;

(c) $\left(\varphi^{x}, \varphi^{y}\right)(x, y, u(x, y))=2 \nabla u(x, y)$ and $\varphi^{z}(x, y, u(x, y))=|\nabla u(x, y)|^{2}$ for every $(x, y) \in \Omega \backslash S_{u}$

(d) $\left(\int_{s}^{t} \varphi^{x}(x, y, z) \mathrm{d} z\right)^{2}+\left(\int_{s}^{t} \varphi^{y}(x, y, z) \mathrm{d} z\right)^{2} \leqslant 1$ for every $(x, y) \in \Omega$ and for every $s, t$ such that $(x, y, s),(x, y, t) \in U$;

(e) $\int_{u^{-}(x, y)}^{u^{+}(x, y)}\left(\varphi^{x}, \varphi^{y}\right)(x, y, z) \mathrm{d} z=v_{u}(x, y)$ for every $(x, y) \in S_{u}$.

The following theorem is proved in [1].

THEOREM 2.1. - If there exists a calibration $\varphi$ for $u$ in $\Omega \times \mathbb{R}$, then $u$ is a Dirichlet minimizer of the Mumford-Shah functional (1.1) in $\Omega$.

What the authors actually prove (but it is not explicitly remarked), is the following more general statement.

THEOREM 2.2. - Let $U$ be an open neighbourhood of $\Gamma_{u}$ such that the intersection with every straight vertical line is connected. If there exists a calibration $\varphi$ for $u$ in $U$, then

$$
\int_{\Omega}|\nabla u(x, y)|^{2} \mathrm{~d} x \mathrm{~d} y+\mathcal{H}^{1}\left(S_{u} \cap \Omega\right) \leqslant \int_{\Omega}|\nabla v(x, y)|^{2} \mathrm{~d} x \mathrm{~d} y+\mathcal{H}^{1}\left(S_{v}\right)
$$

for every $v \in S B V(\Omega)$ such that $v=u$ on $\partial \Omega$ and $\Gamma_{v} \subset U$. 


\section{Proof of Theorem 1.2}

LEMMA 3.1. - Let $U$ be an open subset of $\mathbb{R}^{2}$ and $I, J$ be two real intervals. Let $u: U \times J \rightarrow I$ be a function of class $C^{1}$ such that

- $u(\cdot, \cdot ; s)$ is harmonic for every $s \in J$

- there exists a $C^{1}$ function $t: U \times I \rightarrow J$ such that $u(x, y ; t(x, y ; z))=z$.

Then, if we define in $U \times I$ the vector field

$$
\phi(x, y, z):=\left(2 \nabla u(x, y ; t(x, y ; z)),|\nabla u(x, y ; t(x, y ; z))|^{2}\right),
$$

where $\nabla u(x, y ; t(x, y ; z))$ denotes the gradient of $u$ with respect to the variables $(x, y)$ computed at $(x, y ; t(x, y ; z)), \phi$ is divergence free in $U \times I$.

Proof. - Let us compute the divergence of $\phi$ :

$$
\begin{aligned}
\operatorname{div} \phi(x, y, z)= & 2 \Delta u(x, y ; t(x, y ; z))+2 \partial_{s} \nabla u(x, y ; t(x, y ; z)) \cdot \nabla t(x, y ; z) \\
& +2 \partial_{z} t(x, y ; z) \nabla u(x, y ; t(x, y ; z)) \cdot \partial_{s} \nabla u(x, y ; t(x, y ; z)),
\end{aligned}
$$

where $\Delta u(x, y ; t(x, y ; z))$ denotes the laplacian of $u$ with respect to $(x, y)$ computed at $(x, y ; t(x, y ; z))$, and $\nabla t(x, y ; z)$ denotes the gradient of $t$ with respect to $(x, y)$. By differentiating the identity verified by the function $t$ first with respect to $z$ and with respect to $(x, y)$, we derive that

$$
\begin{aligned}
& \partial_{s} u(x, y ; t(x, y ; z)) \partial_{z} t(x, y ; z)=1, \\
& \nabla u(x, y ; t(x, y ; z))+\partial_{s} u(x, y ; t(x, y ; z)) \nabla t(x, y ; z)=0 .
\end{aligned}
$$

Using these identities and substituting in (3.1), we finally obtain

$$
\operatorname{div} \phi(x, y, z)=2 \Delta u(x, y ; t(x, y ; z))=0,
$$

since by assumption $u$ is harmonic with respect to $(x, y)$.

Proof of Theorem 1.2. - In the sequel, the intersection $\Gamma \cap \bar{\Omega}$ will be still denoted by $\Gamma$. Let

$$
\Gamma:\left\{\begin{array}{l}
x=x(s) \\
y=y(s)
\end{array}\right.
$$

be a parameterization by the arc-length, where $s$ varies in $[0, l(\Gamma)]$; we choose as orientation the normal vector field $v(s)=(-\dot{y}(s), \dot{x}(s))$.

By Cauchy-Kowalevski Theorem (see [7]) there exist an open neighbourhood $U$ of $\Gamma$ contained in $\Omega_{0}$ and a harmonic function $\xi$ defined on $U$ such that

$$
\xi(\Gamma(s))=s \quad \text { and } \quad \frac{\partial \xi}{\partial v}(\Gamma(s))=0
$$

We can suppose that $U$ is simply connected. Let $\eta: U \rightarrow \mathbb{R}^{2}$ be the harmonic conjugate of $\xi$ that vanishes on $\Gamma$, i.e., the function satisfying $\partial_{x} \eta(x, y)=-\partial_{y} \xi(x, y), \partial_{y} \eta(x, y)=$ $\partial_{x} \xi(x, y)$, and $\eta(\Gamma(s))=0$.

Taking $U$ smaller if needed, we can suppose that the map $\Phi(x, y):=(\xi(x, y), \eta(x, y))$ is invertible on $U$. We call $\Psi$ the inverse function $(\xi, \eta) \mapsto(\tilde{x}(\xi, \eta), \tilde{y}(\xi, \eta))$, which 
is defined in the open set $V:=\Phi(U)$. Note that, if $U$ is small enough, then $(\tilde{x}(\xi, \eta), \tilde{y}(\xi, \eta))$ belongs to $\Gamma$ if and only if $\eta=0$. Moreover,

$$
D \Psi=\left(\begin{array}{cc}
\partial_{\xi} \tilde{x} & \partial_{\eta} \tilde{x} \\
\partial_{\xi} \tilde{y} & \partial_{\eta} \tilde{y}
\end{array}\right)=\frac{1}{|\nabla \xi|^{2}}\left(\begin{array}{cc}
\partial_{x} \xi & \partial_{x} \eta \\
\partial_{y} \xi & \partial_{y} \eta
\end{array}\right)
$$

where, in the last formula, all functions are computed at $(x, y)=\Psi(\xi, \eta)$, and so

$$
\partial_{\xi} \tilde{x}=\partial_{\eta} \tilde{y} \quad \text { and } \quad \partial_{\eta} \tilde{x}=-\partial_{\xi} \tilde{y} .
$$

In particular, $\tilde{x}$ and $\tilde{y}$ are harmonic.

On $U$ we will use the coordinate system $(\xi, \eta)$ given by $\Phi$. By (3.2) the canonical basis of the tangent space to $U$ at a point $(x, y)$ is given by

$$
\tau_{\xi}=\frac{\nabla \xi}{|\nabla \xi|^{2}}, \quad \tau_{\eta}=\frac{\nabla \eta}{|\nabla \eta|^{2}} .
$$

For every $(\xi, \eta) \in V$, let $G(\xi, \eta)$ be the matrix associated with the first fundamental form of $U$ in the coordinate system $(\xi, \eta)$, and let $g(\xi, \eta)$ be its determinant. By (3.2) and (3.4),

$$
g=\left(\left(\partial_{\xi} \tilde{x}\right)^{2}+\left(\partial_{\xi} \tilde{y}\right)^{2}\right)^{2}=\frac{1}{|\nabla \xi(\Psi)|^{4}} .
$$

We set $\gamma(\xi, \eta)=\sqrt[4]{g(\xi, \eta)}$.

From now on we will assume that $V$ is symmetric with respect to $\{(\xi, \eta) \in \Phi(U)$ : $\eta=0\}$.

Note that we can write the function $u$ in this new coordinate system as

$$
u(\xi, \eta)= \begin{cases}u_{1}(\xi, \eta) & \text { if }(\xi, \eta) \in V, \eta<0 \\ u_{2}(\xi, \eta) & \text { if }(\xi, \eta) \in V, \eta>0\end{cases}
$$

where we can suppose that $u_{1}$ and $u_{2}$ are defined in $V$ (indeed, $u_{1}$ is a priori defined only on the set $\{(\xi, \eta) \in V: \eta<0\}$, but it can be extended to $V$ by reflection; an analogous argument applies to $\left.u_{2}\right), 0<u_{1}(\xi, 0)<u_{2}(\xi, 0)$ for every $(\xi, 0) \in V$, and

(i) $\partial_{\xi \xi}^{2} u_{i}(\xi, \eta)+\partial_{\eta \eta}^{2} u_{i}(\xi, \eta)=0$ for $i=1,2$;

(ii) $\partial_{\eta} u_{1}(\xi, 0)=\partial_{\eta} u_{2}(\xi, 0)=0$;

(iii) $\left(\partial_{\xi} u_{2}(\xi, 0)\right)^{2}-\left(\partial_{\xi} u_{1}(\xi, 0)\right)^{2}=\operatorname{curv} \Gamma(\xi)$.

The calibration $\varphi(x, y, z)$ on $U \times \mathbb{R}$ will be written as

$$
\varphi(x, y, z)=\frac{1}{\gamma^{2}(\xi(x, y), \eta(x, y))} \phi(\xi(x, y), \eta(x, y), z),
$$

where $\phi: V \times \mathbb{R} \rightarrow \mathbb{R}^{3}$ can be represented by

$$
\phi(\xi, \eta, z)=\phi^{\xi}(\xi, \eta, z) \tau_{\xi}+\phi^{\eta}(\xi, \eta, z) \tau_{\eta}+\phi^{z}(\xi, \eta, z) e_{z},
$$

where $e_{z}$ is the third vector of the canonical basis of $\mathbb{R}^{3}$, and $\tau_{\xi}, \tau_{\eta}$ are computed at the point $\Psi(\xi, \eta)$. We now reformulate the conditions of Section 2 in this new coordinate 
system. It is known from Differential Geometry (see, e.g., [4, Proposition 3.5]) that, if $X=X^{\xi} \tau_{\xi}+X^{\eta} \tau_{\eta}$ is a vector field on $U$, then the divergence of $X$ is given by

$$
\operatorname{div} X=\frac{1}{\gamma^{2}}\left(\partial_{\xi}\left(\gamma^{2} X^{\xi}\right)+\partial_{\eta}\left(\gamma^{2} X^{\eta}\right)\right)
$$

Using (3.4)-(3.7), and (3.8) it turns out that $\varphi$ is a calibration if the following conditions are satisfied:

(a) $\partial_{\xi} \phi^{\xi}+\partial_{\eta} \phi^{\eta}+\partial_{z} \phi^{z}=0$ for every $(\xi, \eta, z) \in V \times \mathbb{R}$;

(b) $\left(\phi^{\xi}(\xi, \eta, z)\right)^{2}+\left(\phi^{\eta}(\xi, \eta, z)\right)^{2} \leqslant 4 \phi^{z}(\xi, \eta, z)$ for every $(\xi, \eta, z) \in V \times \mathbb{R}$;

(c) $\phi^{\xi}(\xi, \eta, u(\xi, \eta))=2 \partial_{\xi} u(\xi, \eta), \phi^{\eta}(\xi, \eta, u(\xi, \eta))=2 \partial_{\eta} u(\xi, \eta)$, and $\phi^{z}(\xi, \eta$, $u(\xi, \eta))=\left(\partial_{\xi} u(\xi, \eta)\right)^{2}+\left(\partial_{\eta} u(\xi, \eta)\right)^{2}$ for every $(\xi, \eta) \in V$

(d) $\left(\int_{s}^{t} \phi^{\xi}(\xi, \eta, z) \mathrm{d} z\right)^{2}+\left(\int_{s}^{t} \phi^{\eta}(\xi, \eta, z) \mathrm{d} z\right)^{2} \leqslant \gamma^{2}(\xi, \eta)$ for every $(\xi, \eta) \in V, s, t \in \mathbb{R}$;

(e) $\int_{u_{1}}^{u_{2}} \phi^{\xi}(\xi, 0, z) \mathrm{d} z=0$ and $\int_{u_{1}}^{u_{2}} \phi^{\eta}(\xi, 0, z) \mathrm{d} z=\gamma(\xi, 0)=1$ for every $(\xi, 0) \in V$.

Given suitable parameters $\varepsilon>0$ and $\lambda>0$, that will be chosen later, we consider the following subsets of $V \times \mathbb{R}$

$$
\begin{aligned}
& A_{1}:=\left\{(\xi, \eta, z) \in V \times \mathbb{R}: z<u_{1}(\xi, \eta)-\varepsilon\right\}, \\
& A_{2}:=\left\{(\xi, \eta, z) \in V \times \mathbb{R}: u_{1}(\xi, \eta)-\varepsilon<z<u_{1}(\xi, \eta)+\varepsilon\right\}, \\
& A_{3}:=\left\{(\xi, \eta, z) \in V \times \mathbb{R}: u_{1}(\xi, \eta)+\varepsilon<z<\beta_{1}(\xi, \eta)\right\}, \\
& A_{4}:=\left\{(\xi, \eta, z) \in V \times \mathbb{R}: \beta_{1}(\xi, \eta)<z<\beta_{2}(\xi, \eta)+1 / \lambda\right\}, \\
& A_{5}:=\left\{(\xi, \eta, z) \in V \times \mathbb{R}: \beta_{2}(\xi, \eta)+1 / \lambda<z<u_{2}(\xi, \eta)-\varepsilon\right\}, \\
& A_{6}:=\left\{(\xi, \eta, z) \in V \times \mathbb{R}: u_{2}(\xi, \eta)-\varepsilon<z<u_{2}(\xi, \eta)+\varepsilon\right\}, \\
& A_{7}:=\left\{(\xi, \eta, z) \in V \times \mathbb{R}: z>u_{2}(\xi, \eta)+\varepsilon\right\},
\end{aligned}
$$

where $\beta_{1}$ and $\beta_{2}$ are suitable smooth functions such that $u_{1}(\xi, 0)<\beta_{1}(\xi, 0)=\beta_{2}(\xi, 0)<$ $u_{2}(\xi, 0)$, which will be defined later. Since we suppose $u_{2}>0$ on $V$, if $\varepsilon$ is small enough, while $\lambda$ is sufficiently large, then the sets $A_{1}, \ldots, A_{7}$ are nonempty and disjoint, provided $V$ is sufficiently small.

The vector $\phi(\xi, \eta, z)$ introduced in (3.6) will be written as

$$
\phi(\xi, \eta, z)=\left(\phi^{\xi \eta}(\xi, \eta, z), \phi^{z}(\xi, \eta, z)\right)
$$

where $\phi^{\xi \eta}$ is the two-dimensional vector given by the pair $\left(\phi^{\xi}, \phi^{\eta}\right)$. For $(\xi, \eta) \in V$ and $z \in \mathbb{R}$ we define $\phi(\xi, \eta, z)$ as follows:

$$
\begin{cases}\left(0, \omega_{1}(\xi, \eta)\right) & \text { in } A_{1} \cup A_{3}, \\ \left(2 \nabla u_{1}-2 \frac{u_{1}-z}{v_{1}} \nabla v_{1},\left|\nabla u_{1}-\frac{u_{1}-z}{v_{1}} \nabla v_{1}\right|^{2}\right) & \text { in } A_{2}, \\ (\lambda \sigma(\xi, \eta) \nabla w, \mu) & \text { in } A_{4}, \\ \left(0, \omega_{2}(\xi, \eta)\right) & \text { in } A_{5} \cup A_{7}, \\ \left(2 \nabla u_{2}-2 \frac{u_{2}-z}{v_{2}} \nabla v_{2},\left|\nabla u_{2}-\frac{u_{2}-z}{v_{2}} \nabla v_{2}\right|^{2}\right) & \text { in } A_{6},\end{cases}
$$


where $\nabla$ denotes the gradient with respect to the variables $(\xi, \eta)$, the functions $v_{i}$ are defined by

$$
v_{1}(\xi, \eta):=\varepsilon+M \eta, \quad v_{2}(\xi, \eta):=\varepsilon-M \eta,
$$

and $M$ and $\mu$ are positive parameters which will be fixed later, while

$$
\omega_{i}(\xi, \eta):=\frac{\varepsilon^{2} M^{2}}{v_{i}^{2}(\xi, \eta)}-\left(\partial_{\xi} u_{i}(\xi, \eta)\right)^{2}-\left(\partial_{\eta} u_{i}(\xi, \eta)\right)^{2}
$$

for $i=1,2$, and for every $(\xi, \eta) \in V$. We choose $w$ as the solution of the Cauchy problem

$$
\left\{\begin{array}{l}
\Delta w=0, \\
w(\xi, 0)=-\frac{2 \varepsilon}{1-2 \varepsilon M} \int_{0}^{\xi} n(s)\left(\partial_{\xi} u_{1}(s, 0)+\partial_{\xi} u_{2}(s, 0)\right) \mathrm{d} s \\
\partial_{\eta} w(\xi, 0)=n(\xi),
\end{array}\right.
$$

where $n$ is a positive analytic function that will be chosen later in a suitable way (if $V$ is sufficiently small, $w$ is defined in $V$ ). To define $\sigma$, we need some further explanations: we call $p(\xi, \eta)$ the solution of the problem

$$
\left\{\begin{array}{l}
\partial_{\eta} p(\xi, \eta)=\frac{\partial_{\xi} w}{\partial_{\eta} w}(p(\xi, \eta), \eta) \\
p(\xi, 0)=\xi
\end{array}\right.
$$

which is defined in $V$, provided $V$ is small enough. By applying the Implicit Function Theorem, it is easy to see that there exists a function $q$ defined in $V$ (take $V$ smaller, if needed) such that

$$
p(q(\xi, \eta), \eta)=\xi
$$

At last, we define

$$
\sigma(\xi, \eta):=\frac{1}{n(q(\xi, \eta))}(1-2 \varepsilon M) .
$$

We choose $\beta_{i}$, for $i=1,2$, as the solution of the Cauchy problem

$$
\left\{\begin{array}{l}
\lambda \sigma(\xi, \eta) \partial_{\xi} w(\xi, \eta) \partial_{\xi} \beta_{i}(\xi, \eta)+\lambda \sigma(\xi, \eta) \partial_{\eta} w(\xi, \eta) \partial_{\eta} \beta_{i}(\xi, \eta)-\mu \\
\quad=-\omega_{i}(\xi, \eta) \\
\beta_{i}(\xi, 0)=\frac{1}{2}\left(u_{1}(\xi, 0)+u_{2}(\xi, 0)\right)
\end{array}\right.
$$

Since the line $\eta=0$ is not characteristic, there exists a unique solution $\beta_{i} \in C^{\infty}(V)$, provided $V$ is small enough.

The purpose of the definition of $\phi$ in $A_{2}$ and $A_{6}$ is to provide a divergence free vector field satisfying condition (c) and such that

$$
\begin{array}{ll}
\phi^{\eta}(\xi, 0, z) \geqslant 0 & \text { for } u_{1}<z<u_{2}, \\
\phi^{\eta}(\xi, 0, z) \leqslant 0 & \text { for } z<u_{1} \text { and } z>u_{2} .
\end{array}
$$


These properties are crucial in order to obtain (d) and (e) simultaneously.

The role of $A_{4}$ is to give the main contribution to the integral in (e). The idea of the construction is to start from the gradient field of a harmonic function $w$ whose normal derivative is positive on the line $\eta=0$, while the tangential derivative is chosen in order to annihilate the $\xi$-component of $\phi$, as required in (e). Then, we multiply the field by a function $\sigma$ which is defined first on $\eta=0$ in order to make (e) true, and then in a neighbourhood of $\eta=0$ by assuming $\sigma$ constant along the integral curves of the gradient field, so that $\sigma \nabla w$ remains divergence free.

The other sets $A_{i}$ are simply regions of transition, where the field is taken purely vertical.

Let us prove condition (a). By Lemma 3.1 it follows that $\phi$ is divergence free in $A_{2} \cup A_{6}$, noting that it is constructed starting from the family of harmonic functions $u_{i}(\xi, \eta)-t v_{i}(\xi, \eta)$.

In $A_{4}$ condition (a) is true since, as remarked above, $\phi$ is the product of $\nabla w$ with the function $\sigma$ which, by construction, is constant along the integral curves of $\nabla w$.

In the other sets, condition (a) is trivially satisfied.

Note that the normal component of $\phi$ is continuous across each $\partial A_{i}$ : for the regions $A_{2}, A_{6}$, and for $A_{4}$, this continuity is guaranteed by our choice of $\omega_{i}$ and $\beta_{i}$ respectively. This implies that (a) is satisfied in the sense of distributions on $V \times \mathbb{R}$.

Since $\omega_{i}(\xi, 0)=M^{2}-\left(\partial_{\xi} u_{i}(\xi, 0)\right)^{2}$, condition (b) is satisfied in $A_{1} \cup A_{3}$ and in $A_{5} \cup A_{7}$ if we require that

$$
M>\sup \left\{\left|\partial_{\xi} u_{i}(\xi, 0)\right|:(\xi, 0) \in V, i=1,2\right\},
$$

provided $V$ is small enough.

Arguing in a similar way, if we impose that

$$
\mu>\sup \left\{\frac{\lambda^{2}}{4}(1-2 \varepsilon M)^{2}\left(1+\frac{4 \varepsilon^{2}}{(1-2 \varepsilon M)^{2}}\left(\partial_{\xi} u_{1}(\xi, 0)+\partial_{\xi} u_{2}(\xi, 0)\right)^{2}\right): \quad(\xi, 0) \in V\right\},
$$

condition (b) holds in $A_{4}$, provided $V$ is sufficiently small.

In the other cases, (b) is trivial.

Looking at the definition of $\phi$ on $A_{2}$ and $A_{6}$, one can check that condition (c) is satisfied.

By direct computations we find that

$$
\begin{aligned}
\int_{u_{1}}^{u_{2}} \phi^{\xi} \mathrm{d} z= & 2 \varepsilon \partial_{\xi} u_{1}+2 \varepsilon \partial_{\xi} u_{2}+\lambda\left(\beta_{2}-\beta_{1}+\frac{1}{\lambda}\right) \sigma \partial_{\xi} w, \\
\int_{u_{1}}^{u_{2}} \phi^{\eta} \mathrm{d} z= & 2 \varepsilon \partial_{\eta} u_{1}+2 \varepsilon \partial_{\eta} u_{2}+M \frac{\varepsilon^{2}}{\varepsilon+M \eta}+M \frac{\varepsilon^{2}}{\varepsilon-M \eta} \\
& +\lambda\left(\beta_{2}-\beta_{1}+\frac{1}{\lambda}\right) \sigma \partial_{\eta} w,
\end{aligned}
$$


for every $(\xi, \eta) \in V$.

By using (3.10) and the definition of $\sigma$, we obtain

$$
\int_{u_{1}(\xi, 0)}^{u_{2}(\xi, 0)} \phi^{\xi}(\xi, 0, z) \mathrm{d} z=0
$$

and

$$
\int_{u_{1}(\xi, 0)}^{u_{2}(\xi, 0)} \phi^{\eta}(\xi, 0, z) \mathrm{d} z=1,
$$

so condition (e) is satisfied.

The proof of condition (d) will be split in two steps: we first prove that condition (d) holds if $s$ and $t$ respectively belong to a suitable neighbourhood of $u_{1}(\xi, \eta)$ and $u_{2}(\xi, \eta)$, whose width is uniform with respect to $(\xi, \eta)$ in $V$; then, by a quite simple continuity argument we show that condition (d) is true if $s$ or $t$ is not too close to $u_{1}(\xi, \eta)$ or $u_{2}(\xi, \eta)$ respectively.

For $(\xi, \eta) \in V$ and $s, t \in \mathbb{R}$, we set

$$
I(\xi, \eta, s, t):=\int_{s}^{t} \phi^{\xi \eta}(\xi, \eta, z) \mathrm{d} z
$$

and we denote by $I^{\xi}$ and $I^{\eta}$ its components.

Step 1. For a suitable choice of $\varepsilon$ and of the function $n$ (see (3.10)) there exists $\delta>0$ such that condition (d) holds for $\left|s-u_{1}(\xi, \eta)\right|<\delta,\left|t-u_{2}(\xi, \eta)\right|<\delta$, and $(\xi, \eta) \in V$, provided $V$ is small enough.

To estimate the vector whose components are given by (3.14) and (3.15), we use suitable polar coordinates. If $V$ is small enough, for every $(\xi, \eta) \in V$ there exist $\rho_{\varepsilon, n}(\xi, \eta)>0$ and $-\pi / 2<\theta_{\varepsilon, n}(\xi, \eta)<\pi / 2$ such that

$$
\begin{aligned}
& I^{\xi}\left(\xi, \eta, u_{1}(\xi, \eta), u_{2}(\xi, \eta)\right)=\rho_{\varepsilon, n}(\xi, \eta) \sin \theta_{\varepsilon, n}(\xi, \eta), \\
& I^{\eta}\left(\xi, \eta, u_{1}(\xi, \eta), u_{2}(\xi, \eta)\right)=\rho_{\varepsilon, n}(\xi, \eta) \cos \theta_{\varepsilon, n}(\xi, \eta) .
\end{aligned}
$$

In the notation above we have made explicit the dependence on the parameter $\varepsilon$ and on the function $n$ which appears in the definition of $w$ (see (3.10)).

In order to prove condition (d), we want to compare the behaviour of the functions $\rho_{\varepsilon, n}$ and $\gamma$ for $|\eta|$ small. We have already proved that $\rho_{\varepsilon, n}(\xi, 0)=\gamma(\xi, 0)=1$; we start computing the first derivative of $\gamma$ and of $\rho_{\varepsilon, n}$ with respect to the variable $\eta$.

CLAIM 1. - There holds that $\partial_{\eta}\left(\left|\nabla_{x y} \xi(\Psi)\right|^{2}\right)(\xi, 0)=-2 \operatorname{curv} \Gamma(\xi)$. 
Proof of the Claim. - By (3.5) we obtain

$$
\left|\nabla_{x y} \xi(\Psi)\right|^{2}=\frac{1}{\left(\partial_{\xi} \tilde{x}\right)^{2}+\left(\partial_{\xi} \tilde{y}\right)^{2}},
$$

hence

$$
\partial_{\eta}\left(\left|\nabla_{x y} \xi(\Psi)\right|\right)^{2}=-\left[\left(\partial_{\xi} \tilde{x}\right)^{2}+\left(\partial_{\xi} \tilde{y}\right)^{2}\right]^{-2}\left(2 \partial_{\xi} \tilde{x} \partial_{\xi \eta}^{2} \tilde{x}+2 \partial_{\xi} \tilde{y} \partial_{\xi \eta}^{2} \tilde{y}\right)
$$

Using the fact that $\left(\partial_{\xi} \tilde{x}\right)^{2}+\left(\partial_{\xi} \tilde{y}\right)^{2}$ is equal to 1 at $(\xi, 0)$, and the equalities in (3.3), we finally get

$$
\partial_{\eta}\left(\left|\nabla_{x y} \xi(\Psi)\right|^{2}\right)(\xi, 0)=-2\left(-\partial_{\xi} \tilde{x} \partial_{\xi \xi}^{2} \tilde{y}+\partial_{\xi} \tilde{y} \partial_{\xi \xi}^{2} \tilde{x}\right)=-2 \operatorname{curv} \Gamma(\xi),
$$

where the last equality follows from (2.1): therefore the claim is proved.

Since $\gamma=\left(\left|\nabla_{x y} \xi(\Psi)\right|^{2}\right)^{-1 / 2}$, one has that $\partial_{\eta} \gamma=-\frac{1}{2}\left(\left|\nabla_{x y} \xi(\Psi)\right|^{2}\right)^{-3 / 2} \partial_{\eta}\left(\left|\nabla_{x y} \xi(\Psi)\right|^{2}\right)$; using the previous claim we can conclude that

$$
\partial_{\eta}(\gamma)(\xi, 0)=-\frac{1}{2} \partial_{\eta}\left(\left|\nabla_{x y} \xi(\Psi)\right|^{2}\right)(\xi, 0)=\operatorname{curv} \Gamma(\xi) .
$$

Using the equality

$$
\rho_{\varepsilon, n}^{2}(\xi, \eta)=\left(I^{\xi}\left(\xi, \eta, u_{1}(\xi, \eta), u_{2}(\xi, \eta)\right)\right)^{2}+\left(I^{\eta}\left(\xi, \eta, u_{1}(\xi, \eta), u_{2}(\xi, \eta)\right)\right)^{2},
$$

we obtain

$$
\begin{aligned}
\partial_{\eta}\left(\rho_{\varepsilon, n}\right)= & \frac{1}{\rho_{\varepsilon, n}} \partial_{\eta}\left(I^{\xi}\left(\xi, \eta, u_{1}, u_{2}\right)\right) I^{\xi}\left(\xi, \eta, u_{1}, u_{2}\right) \\
& +\frac{1}{\rho_{\varepsilon, n}} \partial_{\eta}\left(I^{\eta}\left(\xi, \eta, u_{1}, u_{2}\right)\right) I^{\eta}\left(\xi, \eta, u_{1}, u_{2}\right) .
\end{aligned}
$$

By (3.16) it follows that the first addend in the expression above is equal to zero at $(\xi, 0)$, while by (3.17) it turns out that $I^{\eta}\left(\xi, 0, u_{1}, u_{2}\right)=\rho_{\varepsilon, n}(\xi, 0)=1$; therefore,

$$
\partial_{\eta}\left(\rho_{\varepsilon, n}\right)(\xi, 0)=\partial_{\eta}\left(I^{\eta}\left(\xi, 0, u_{1}, u_{2}\right)\right) \text {. }
$$

By (3.15) it follows that

$$
\begin{aligned}
\partial_{\eta}\left(I^{\eta}\left(\xi, \eta, u_{1}, u_{2}\right)\right)= & 2 \varepsilon \partial_{\eta}^{2} u_{1}+2 \varepsilon \partial_{\eta}^{2} u_{2}-\frac{\varepsilon^{2}}{(\varepsilon+M \eta)^{2}} M^{2}+\frac{\varepsilon^{2}}{(\varepsilon-M \eta)^{2}} M^{2} \\
& +\lambda\left(\partial_{\eta} \beta_{2}-\partial_{\eta} \beta_{1}\right) \sigma \partial_{\eta} w+\lambda\left(\beta_{2}-\beta_{1}+1 / \lambda\right) \partial_{\eta}\left(\sigma \partial_{\eta} w\right) .
\end{aligned}
$$

From (3.13) and the Euler condition (iii), we have that

$$
\begin{aligned}
\lambda\left(\partial_{\eta} \beta_{2}(\xi, 0)-\partial_{\eta} \beta_{1}(\xi, 0)\right) \sigma(\xi, 0) \partial_{\eta} w(\xi, 0) & =-\omega_{2}(\xi, 0)+\omega_{1}(\xi, 0) \\
& =\left(\partial_{\xi} u_{2}(\xi, 0)\right)^{2}-\left(\partial_{\xi} u_{1}(\xi, 0)\right)^{2} \\
& =\operatorname{curv} \Gamma(\xi),
\end{aligned}
$$


while

$$
\partial_{\eta}\left(\sigma \partial_{\eta} w\right)(\xi, 0)=-\partial_{\xi}\left(\sigma \partial_{\xi} w\right)(\xi, 0)=\partial_{\xi}\left(2 \varepsilon \partial_{\xi} u_{1}(\xi, 0)+2 \varepsilon \partial_{\xi} u_{2}\right)(\xi, 0),
$$

where we have used the fact that $\sigma \nabla w$ is divergence free and the definition of $\sigma$ and $w$. Putting this last fact together with (3.22), (3.23), and the harmonicity of $u_{i}$, we finally get

$$
\partial_{\eta}\left(\rho_{\varepsilon, n}\right)(\xi, 0)=\operatorname{curv} \Gamma(\xi)=\partial_{\eta}(\gamma)(\xi, 0) .
$$

CLAIM 2. - There holds that $\partial_{\eta \eta}^{2}\left(\left|\nabla_{x y} \xi(\Psi)\right|^{2}\right)(\xi, 0)=4[\operatorname{curv} \Gamma(\xi)]^{2}$.

Proof of the Claim. - By differentiating with respect to $\eta$ the expression in (3.20) and by (3.3), we obtain

$$
\begin{aligned}
\partial_{\eta \eta}^{2}\left(\left|\nabla_{x y} \xi(\Psi)\right|^{2}\right)= & -2\left[\left(\partial_{\xi} \tilde{x}\right)^{2}+\left(\partial_{\xi} \tilde{y}\right)^{2}\right]^{-2}\left[\left(\partial_{\xi \eta} \tilde{x}\right)^{2}+\partial_{\xi} \tilde{x} \partial_{\xi \eta \eta}^{3} \tilde{x}+\left(\partial_{\xi \eta} \tilde{y}\right)^{2}+\partial_{\xi} \tilde{y} \partial_{\xi \eta \eta}^{3} \tilde{y}\right] \\
& +8\left[\left(\partial_{\xi} \tilde{x}\right)^{2}+\left(\partial_{\xi} \tilde{y}\right)^{2}\right]^{-3}\left(\partial_{\xi} \tilde{x} \partial_{\xi \eta}^{2} \tilde{x}+\partial_{\xi} \tilde{y} \partial_{\xi \eta}^{2} \tilde{y}\right)^{2} \\
= & -2\left[\left(\partial_{\xi} \tilde{x}\right)^{2}+\left(\partial_{\xi} \tilde{y}\right)^{2}\right]^{-2}\left[\left(\partial_{\xi \xi}^{2} \tilde{y}\right)^{2}+\left(\partial_{\xi \xi}^{2} \tilde{x}\right)^{2}-\partial_{\xi} \tilde{x} \partial_{\xi \xi \xi}^{3} \tilde{x}-\partial_{\xi} \tilde{y} \partial_{\xi \xi \xi}^{3} \tilde{y}\right] \\
& +8\left[\left(\partial_{\xi} \tilde{x}\right)^{2}+\left(\partial_{\xi} \tilde{y}\right)^{2}\right]^{-3}\left(-\partial_{\xi} \tilde{x} \partial_{\xi \xi}^{2} \tilde{y}+\partial_{\xi} \tilde{y} \partial_{\xi \xi}^{2} \tilde{x}\right)^{2} .
\end{aligned}
$$

Note that

$$
-\partial_{\xi} \tilde{x} \partial_{\xi \xi \xi}^{3} \tilde{x}-\partial_{\xi} \tilde{y} \partial_{\xi \xi \xi}^{3} \tilde{y}=\left(\partial_{\xi \xi}^{2} \tilde{y}\right)^{2}+\left(\partial_{\xi \xi}^{2} \tilde{x}\right)^{2}-\frac{1}{2} \partial_{\xi \xi}^{2}\left(\left(\partial_{\xi} \tilde{x}\right)^{2}+\left(\partial_{\xi} \tilde{y}\right)^{2}\right) .
$$

Using (2.1), (2.2), and the fact that $\left(\partial_{\xi} \tilde{x}\right)^{2}+\left(\partial_{\xi} \tilde{y}\right)^{2}$ is equal to 1 at $(\xi, 0)$, we obtain the claim.

By using Claims 1 and 2, we can conclude that

$$
\begin{aligned}
\partial_{\eta \eta}^{2}(\gamma)(\xi, 0)= & {\left[\frac{3}{4}\left(\left|\nabla_{x y} \xi(\Psi)\right|^{2}\right)^{-5 / 2}\left[\partial_{\eta}\left(\left|\nabla_{x y} \xi(\Psi)\right|^{2}\right)\right]^{2}\right.} \\
& \left.-\frac{1}{2}\left(\left|\nabla_{x y} \xi(\Psi)\right|^{2}\right)^{-3 / 2} \partial_{\eta \eta}^{2}\left(\left|\nabla_{x y} \xi(\Psi)\right|^{2}\right)\right]\left.\right|_{(\xi, 0)} \\
= & {[\operatorname{curv} \Gamma(\xi)]^{2} . }
\end{aligned}
$$

The second derivative of $\rho_{\varepsilon, n}$ with respect to $\eta$ is given by

$$
\begin{aligned}
\partial_{\eta \eta}^{2}\left(\rho_{\varepsilon, n}\right)= & \frac{1}{\rho_{\varepsilon, n}}\left\{\left[\partial_{\eta}\left(I^{\xi}\left(\xi, \eta, u_{1}, u_{2}\right)\right)\right]^{2}+\partial_{\eta \eta}^{2}\left(I^{\xi}\left(\xi, \eta, u_{1}, u_{2}\right)\right) I^{\xi}\left(\xi, \eta, u_{1}, u_{2}\right)\right. \\
& \left.+\left[\partial_{\eta}\left(I^{\eta}\left(\xi, \eta, u_{1}, u_{2}\right)\right)\right]^{2}+\partial_{\eta \eta}^{2}\left(I^{\eta}\left(\xi, \eta, u_{1}, u_{2}\right)\right) I^{\eta}\left(\xi, \eta, u_{1}, u_{2}\right)\right\} \\
& -\frac{1}{\rho_{\varepsilon, n}}\left[\partial_{\eta}\left(\rho_{\varepsilon, n}\right)\right]^{2} .
\end{aligned}
$$

By equalities (3.16), (3.17), and (3.21), the expression above computed at $(\xi, 0)$ reduces to

$$
\partial_{\eta \eta}^{2}\left(\rho_{\varepsilon, n}\right)(\xi, 0)=\left[\left.\partial_{\eta}\left(I^{\xi}\left(\xi, \eta, u_{1}, u_{2}\right)\right)\right|_{(\xi, 0)}\right]^{2}+\left.\partial_{\eta \eta}^{2}\left(I^{\eta}\left(\xi, \eta, u_{1}, u_{2}\right)\right)\right|_{(\xi, 0)} .
$$


By differentiating (3.14) and (3.22) with respect to $\eta$, we obtain that

$$
\partial_{\eta}\left(I^{\xi}\left(\xi, \eta, u_{1}, u_{2}\right)\right)(\xi, 0)=\left.\left[\lambda\left(\partial_{\eta} \beta_{2}-\partial_{\eta} \beta_{1}\right) \sigma \partial_{\xi} w+\partial_{\eta} \sigma \partial_{\xi} w+\sigma \partial_{\xi \eta}^{2} w\right]\right|_{(\xi, 0)},
$$

and

$$
\begin{aligned}
\partial_{\eta \eta}^{2}\left(I^{\eta}\left(\xi, \eta, u_{1}, u_{2}\right)\right)(\xi, 0)= & \frac{4}{\varepsilon} M^{3}+\lambda\left[\partial_{\eta \eta}^{2} \beta_{2}(\xi, 0)-\partial_{\eta \eta}^{2} \beta_{1}(\xi, 0)\right] \sigma(\xi, 0) \partial_{\eta} w(\xi, 0) \\
& +2 \lambda\left[\partial_{\eta} \beta_{2}(\xi, 0)-\partial_{\eta} \beta_{1}(\xi, 0)\right] \partial_{\eta}\left(\sigma \partial_{\eta} w\right)(\xi, 0) \\
& +\partial_{\eta \eta}^{2} \sigma(\xi, 0) \partial_{\eta} w(\xi, 0)+2 \partial_{\eta} \sigma(\xi, 0) \partial_{\eta \eta}^{2} w(\xi, 0) \\
& +\sigma(\xi, 0) \partial_{\eta \eta \eta}^{3} w(\xi, 0)
\end{aligned}
$$

while, by using Eq. (3.13),

$$
\begin{aligned}
& {\left.\left[\lambda\left(\partial_{\eta \eta}^{2} \beta_{2}-\partial_{\eta \eta}^{2} \beta_{1}\right) \sigma \partial_{\eta} w\right]\right|_{(\xi, 0)}} \\
& \quad=\left.\left[\partial_{\eta} \omega_{1}-\partial_{\eta} \omega_{2}-\lambda \partial_{\eta}\left(\partial_{\xi} \beta_{2}-\partial_{\xi} \beta_{1}\right) \sigma \partial_{\xi} w-\lambda \partial_{\eta}\left(\sigma \partial_{\eta} w\right)\left(\partial_{\eta} \beta_{2}-\partial_{\eta} \beta_{1}\right)\right]\right|_{(\xi, 0)} \\
& \quad=\left.\left[-\frac{4}{\varepsilon} M^{3}-\lambda \partial_{\xi}\left(\partial_{\eta} \beta_{2}-\partial_{\eta} \beta_{1}\right) \sigma \partial_{\xi} w+\lambda \partial_{\xi}\left(\sigma \partial_{\xi} w\right)\left(\partial_{\eta} \beta_{2}-\partial_{\eta} \beta_{1}\right)\right]\right|_{(\xi, 0)} .
\end{aligned}
$$

Since by (3.23) and by the definition of $\sigma$ we have that

$$
\lambda\left[\partial_{\eta} \beta_{2}(\xi, 0)-\partial_{\eta} \beta_{1}(\xi, 0)\right]=\frac{\operatorname{curv} \Gamma(\xi)}{1-2 \varepsilon M},
$$

and moreover,

$$
\sigma(\xi, 0) \partial_{\xi} w(\xi, 0)=-2 \varepsilon\left(\partial_{\xi} u_{1}(\xi, 0)+\partial_{\xi} u_{2}(\xi, 0)\right)
$$

we obtain that

$$
\begin{gathered}
{\left.\left[\lambda\left(\partial_{\eta \eta}^{2} \beta_{2}-\partial_{\eta \eta}^{2} \beta_{1}\right) \sigma \partial_{\eta} w+2 \lambda\left(\partial_{\eta} \beta_{2}-\partial_{\eta} \beta_{1}\right) \partial_{\eta}\left(\sigma \partial_{\eta} w\right)\right]\right|_{(\xi, 0)}} \\
\quad=-\frac{4}{\varepsilon} M^{3}+\frac{2 \varepsilon}{1-2 \varepsilon M} \partial_{\xi}\left(\left(\partial_{\xi} u_{1}-\partial_{\xi} u_{2}\right) \operatorname{curv} \Gamma\right)(\xi, 0) .
\end{gathered}
$$

By using the definition of $\sigma$, we can write

$$
\begin{aligned}
\partial_{\eta} \sigma & =-(1-2 \varepsilon M) \frac{n^{\prime}(\xi)}{n^{2}(\xi)} \partial_{\eta} q, \\
\partial_{\eta \eta}^{2} \sigma & =-(1-2 \varepsilon M)\left[-2 \frac{\left(n^{\prime}(\xi)\right)^{2}}{n^{3}(\xi)}\left(\partial_{\eta} q\right)^{2}+\frac{n^{\prime \prime}(\xi)}{n^{2}(\xi)}\left(\partial_{\eta} q\right)^{2}+\frac{n^{\prime}(\xi)}{n^{2}(\xi)} \partial_{\eta}^{2} q\right] .
\end{aligned}
$$

In order to compute the derivatives of $q$, we differentiate equality (3.12) with respect to $\eta$ :

$$
\begin{aligned}
\partial_{\eta} q(\xi, 0) & =-\partial_{\eta} p(\xi, 0)=\frac{2 \varepsilon}{1-2 \varepsilon M}\left(\partial_{\xi} u_{1}(\xi, 0)+\partial_{\xi} u_{2}(\xi, 0)\right), \\
\partial_{\eta \eta}^{2} q(\xi, 0) & =-2 \partial_{\xi \eta}^{2} p(\xi, 0) \partial_{\eta} q(\xi, 0)-\partial_{\eta \eta}^{2} p(\xi, 0) \\
& =\left[-\frac{\left(\partial_{\xi} w\right)^{2}}{\left(\partial_{\eta} w\right)^{3}} \partial_{\xi \eta}^{2} w-\frac{1}{\partial_{\eta} w} \partial_{\xi \eta}^{2} w\right](\xi, 0) .
\end{aligned}
$$


By the definition of $w$, we obtain

$$
\partial_{\eta}^{2} q(\xi, 0)=-\frac{n^{\prime}(\xi)}{n(\xi)}-\frac{n^{\prime}(\xi)}{n(\xi)} \frac{4 \varepsilon^{2}}{(1-2 \varepsilon M)^{2}}\left(\partial_{\xi} u_{1}(\xi, 0)+\partial_{\xi} u_{2}(\xi, 0)\right)^{2} .
$$

Finally, we have

$$
\begin{aligned}
& \partial_{\eta \eta}^{2} w(\xi, 0)=-\partial_{\xi \xi}^{2} w(\xi, 0)=\left.\frac{2 \varepsilon}{1-2 \varepsilon M}\left[n^{\prime}\left(\partial_{\xi} u_{1}+\partial_{\xi} u_{2}\right)+n\left(\partial_{\xi \xi}^{2} u_{1}+\partial_{\xi \xi}^{2} u_{2}\right)\right]\right|_{(\xi, 0)}, \\
& \partial_{\eta \eta \eta}^{3} w(\xi, 0)=-\partial_{\xi \xi}^{2} \partial_{\eta} w(\xi, 0)=-n^{\prime \prime}(\xi) .
\end{aligned}
$$

By substituting all information above in (3.27) and in (3.28), and by using (3.26), we finally obtain that

$$
\begin{aligned}
\partial_{\eta \eta}^{2}\left(\rho_{\varepsilon, n}\right)(\xi, 0) & =-a_{\varepsilon}(\xi) \frac{n^{\prime \prime}(\xi)}{n(\xi)}+h_{\varepsilon}\left(\xi, \frac{n^{\prime}(\xi)}{n(\xi)}\right) \\
& =-a_{\varepsilon}(\xi)\left(\frac{n^{\prime}(\xi)}{n(\xi)}\right)^{\prime}+h_{\varepsilon}\left(\xi, \frac{n^{\prime}(\xi)}{n(\xi)}\right)-a_{\varepsilon}(\xi)\left(\frac{n^{\prime}(\xi)}{n(\xi)}\right)^{2},
\end{aligned}
$$

where

$$
\begin{gathered}
a_{\varepsilon}(\xi) \rightarrow 1 \quad \text { uniformly in }[0, l(\Gamma)], \\
h_{\varepsilon}(\xi, \tau) \rightarrow 2 \tau^{2} \quad \text { uniformly on the compact sets of }[0, l(\Gamma)] \times \mathbb{R},
\end{gathered}
$$

as $\varepsilon \rightarrow 0$.

CLAim 3. - There exists $\bar{\varepsilon}>0$ such that for every $\varepsilon \in(0, \bar{\varepsilon})$, we can find an analytic function $n:[0, l(\Gamma)] \rightarrow(0,+\infty)$ satisfying

$$
\partial_{\eta \eta}^{2}\left(\rho_{\varepsilon, n}-\gamma\right)(\xi, 0)=-\frac{\pi^{2}}{16 l^{2}(\Gamma)} \quad \text { and } \quad\left|\frac{n^{\prime}(\xi)}{n(\xi)}\right| \leqslant N \quad \forall \xi \in[0, l(\Gamma)]
$$

where $N:=1+\max \left\{\frac{\pi}{4 l(\Gamma)}, k(\Gamma)\right\}$ and $k(\Gamma)=\|\operatorname{curv} \Gamma\|_{\infty}$.

Proof of the Claim. - Set $\tau:=n^{\prime} / n$; in order to prove the claim, by (3.29) and (3.25) we study the Cauchy problem

$$
\left\{\begin{array}{l}
-a_{\varepsilon}(\xi) \tau^{\prime}+h_{\varepsilon}(\xi, \tau)-\tau^{2}-[\operatorname{curv} \Gamma(\xi)]^{2}=-\frac{\pi^{2}}{16 l^{2}(\Gamma)}, \\
\tau(0)=0
\end{array}\right.
$$

and we investigate for which values of $\varepsilon$ it admits a solution defined in the whole interval $[0, l(\Gamma)]$, with $L^{\infty}$-norm less than $N$. As $\varepsilon \rightarrow 0$, by (3.30) we obtain the limit problem

$$
\left\{\begin{array}{l}
-\tau^{\prime}+\tau^{2}-(\operatorname{curv} \Gamma)^{2}=-\frac{\pi^{2}}{16 l^{2}(\Gamma)}, \\
\tau(0)=0
\end{array}\right.
$$


By comparing with the solutions $\tau_{1}$ and $\tau_{2}$ of the Cauchy problems

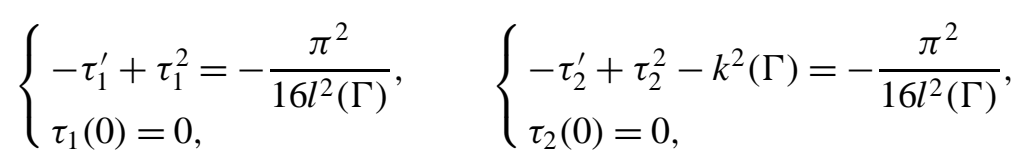

one easily sees that the solution of (3.33) is defined in $[0, l(\Gamma)]$, with $L^{\infty}$-norm less than the maximum between $\left\|\tau_{1}\right\|_{\infty}$ and $\left\|\tau_{2}\right\|_{\infty}$, which is, by explicit computation, less than $\max \{\pi /(4 l(\Gamma)), k(\Gamma)\}$. By the theorem of continuous dependence on the coefficients (see [6]), we can find $\bar{\varepsilon}$ such that, for every $\varepsilon \in(0, \bar{\varepsilon})$, the solution of (3.32) is defined in $[0, l(\Gamma)]$ with $L^{\infty}$-norm less than $N$.

For every $\varepsilon \in(0, \bar{\varepsilon})$, we set

$$
n_{\varepsilon}(\xi):=\mathrm{e}^{\int_{0}^{\xi} \tau_{\varepsilon}(s) \mathrm{d} s},
$$

where $\tau_{\varepsilon}$ is the solution of (3.32).

From now on we will simply write $\rho_{\varepsilon}$ and $\theta_{\varepsilon}$ instead of $\rho_{\varepsilon, n_{\varepsilon}}$ and $\theta_{\varepsilon, n_{\varepsilon}}$.

We now want to estimate the angle $\theta_{\varepsilon}(\xi, \eta)$ by a quantity which is independent of $\varepsilon$. Since by (3.14) and (3.15)

$\tan \theta_{\varepsilon}$

$$
=\frac{2 \varepsilon \partial_{\xi} u_{1}+2 \varepsilon \partial_{\xi} u_{2}+\lambda\left(\beta_{2}-\beta_{1}+\frac{1}{\lambda}\right) \sigma \partial_{\xi} w}{2 \varepsilon \partial_{\eta} u_{1}+2 \varepsilon \partial_{\eta} u_{2}+M \varepsilon^{2}(\varepsilon+M \eta)^{-1}+M \varepsilon^{2}(\varepsilon-M \eta)^{-1}+\lambda\left(\beta_{2}-\beta_{1}+\frac{2}{\lambda}\right) \sigma \partial_{\eta} w},
$$

we have

$\partial_{\eta} \theta_{\varepsilon}(\xi, 0)$

$$
=-\frac{2 \varepsilon}{1-2 \varepsilon M}\left(\partial_{\xi} u_{1}+\partial_{\xi} u_{2}\right)\left(\operatorname{curv} \Gamma-2 \varepsilon\left(\partial_{\xi} u_{1}+\partial_{\xi} u_{2}\right) \frac{n_{\varepsilon}^{\prime}(\xi)}{n_{\varepsilon}(\xi)}\right)+(1-2 \varepsilon M) \frac{n_{\varepsilon}^{\prime}(\xi)}{n_{\varepsilon}(\xi)},
$$

and so, by Claim 3, if $\varepsilon$ is sufficiently small,

$$
\left|\partial_{\eta} \theta_{\varepsilon}(\xi, 0)\right|<N \quad \forall \xi \in[0, l(\Gamma)]
$$

Let $\tilde{\theta}(\eta)$ be an arbitrary continuous function with

$$
\tilde{\theta}(0)=0 \quad \text { and } \quad \tilde{\theta}^{\prime}(0)=N
$$

by (3.36), it follows that

$$
\left|\theta_{\varepsilon}(\xi, \eta)\right|<\tilde{\theta}(\eta) \operatorname{sign} \eta
$$

for every $(\xi, \eta) \in V$, provided $V$ is sufficiently small.

Given $h>0$, we consider the vectors

$$
\begin{aligned}
& b_{1}^{h}(\xi, \eta, s):=\left(0,-2\left(s-u_{1}(\xi, \eta)\right) \partial_{\eta} u_{1}(\xi, \eta)-h\left(s-u_{1}(\xi, \eta)\right)^{2}\right), \\
& b_{2}^{h}(\xi, \eta, t):=\left(0,2\left(t-u_{2}(\xi, \eta)\right) \partial_{\eta} u_{2}(\xi, \eta)-h\left(t-u_{2}(\xi, \eta)\right)^{2}\right)
\end{aligned}
$$


for $(\xi, \eta) \in V$ and $s, t \in \mathbb{R}$. We denote by $B(r)$ the open ball centred at $(0,-r)$ with radius $r$.

Let us define $r_{\varepsilon}^{h}(\xi, \eta, s, t)$ as the maximum radius $r$ such that the set

$$
\left(\rho_{\varepsilon}(\xi, \eta) \sin \tilde{\theta}(\eta), \rho_{\varepsilon}(\xi, \eta) \cos \tilde{\theta}(\eta)\right)+b_{1}^{h}(\xi, \eta, s)+b_{2}^{h}(\xi, \eta, t)+B(r)
$$

is contained in the ball centred at $(0,0)$ with radius $\gamma(\xi, \eta)$.

Claim 4. - If we define

$$
d:=\frac{1}{1+16 l^{2}(\Gamma) N^{2} / \pi^{2}},
$$

where $N$ is the constant introduced in the previous claim, then there exists $h>0$ such that for every $\varepsilon \in(0, \bar{\varepsilon})$ (see Claim 3$)$, there exists $\delta \in(0, \varepsilon)$ so that, if $V$ is small enough,

$$
\inf \left\{2 r_{\varepsilon}^{h}(\xi, \eta, s, t):(\xi, \eta) \in V,\left|s-u_{1}(\xi, \eta)\right| \leqslant \delta,\left|t-u_{2}(\xi, \eta)\right| \leqslant \delta\right\}>\frac{d}{2} .
$$

Proof of the Claim. - Let $\bar{\rho}_{\varepsilon}^{h}(\xi, \eta, s, t)>0$ and $-\pi / 2<\bar{\theta}_{\varepsilon}^{h}(\xi, \eta, s, t)<\pi / 2$ be such that

$$
\begin{aligned}
& \left(\rho_{\varepsilon}(\xi, \eta) \sin \tilde{\theta}(\eta), \rho_{\varepsilon}(\xi, \eta) \cos \tilde{\theta}(\eta)\right)+b_{1}^{h}(\xi, \eta, s)+b_{2}^{h}(\xi, \eta, t) \\
& \quad=\left(\bar{\rho}_{\varepsilon}^{h}(\xi, \eta, s, t) \sin \bar{\theta}_{\varepsilon}^{h}(\xi, \eta, s, t), \bar{\rho}_{\varepsilon}^{h}(\xi, \eta, s, t) \cos \bar{\theta}_{\varepsilon}^{h}(\xi, \eta, s, t)\right) .
\end{aligned}
$$

To prove Claim 4 , it is enough to show that, for every $\varepsilon \in(0, \bar{\varepsilon})$, there exists $\delta \in(0, \varepsilon)$ with the property that

$$
\left(1-\frac{d}{2} \cos \bar{\theta}_{\varepsilon}^{h}(\xi, \eta, s, t)\right) \bar{\rho}_{\varepsilon}^{h}(\xi, \eta, s, t)<\left(1-\frac{d}{2}\right) \gamma(\xi, \eta)
$$

for $\left|s-u_{1}(\xi, \eta)\right| \leqslant \delta,\left|t-u_{2}(\xi, \eta)\right| \leqslant \delta$, and $(\xi, \eta) \in V$ with $\eta \neq 0$, provided $V$ is sufficiently small. Indeed, if (3.42) holds, it follows in particular that $\bar{\rho}_{\varepsilon}^{h}(\xi, \eta, s, t)<$ $\gamma(\xi, \eta)$, and this inequality with some easy geometric computations implies that

$$
2 r_{\varepsilon}^{h}(\xi, \eta, s, t)=\frac{\gamma^{2}(\xi, \eta)-\left(\bar{\rho}_{\varepsilon}^{h}(\xi, \eta, s, t)\right)^{2}}{\gamma-\bar{\rho}_{\varepsilon}^{h}(\xi, \eta, s, t) \cos \bar{\theta}_{\varepsilon}^{h}(\xi, \eta, s, t)} ;
$$

at this point, it is easy to see that, if $V$ is small enough, inequality (3.42) implies that $2 r_{\varepsilon}^{h}(\xi, \eta, s, t)>d / 2$, that is Claim 4. So let us prove (3.42).

We set

$$
f^{d, h}(\xi, \eta, s, t):=\left(1-\frac{d}{2} \cos \bar{\theta}_{\varepsilon}^{h}(\xi, \eta, s, t)\right) \bar{\rho}_{\varepsilon}^{h}(\xi, \eta, s, t)-\left(1-\frac{d}{2}\right) \gamma(\xi, \eta)
$$

and we note that $f^{d, h}\left(\xi, 0, u_{1}(\xi, 0), u_{2}(\xi, 0)\right)=0$. We will show that

1. $\nabla_{\eta s t} f^{d, h}\left(\xi, 0, u_{1}(\xi, 0), u_{2}(\xi, 0)\right)=0$ if $(\xi, 0) \in V$,

2. $\nabla_{\eta s t}^{2} f^{d, h}\left(\xi, 0, u_{1}(\xi, 0), u_{2}(\xi, 0)\right)$ is negative definite if $(\xi, 0) \in V$, 
where $\nabla_{\eta s t} f^{d, h}$ and $\nabla_{\eta s t}^{2} f^{d, h}$ denote respectively the gradient and the hessian matrix of $f^{d, h}$ with respect to the variables $(\eta, s, t)$. Equality 1 follows by direct computations and by (3.24). Using (3.41), the equality in (3.31), and (3.37), we obtain

$$
\partial_{\eta \eta}^{2} f^{d, h}\left(\xi, 0, u_{1}(\xi, 0), u_{2}(\xi, 0)\right)=-\frac{\pi^{2}}{16 l^{2}(\Gamma)}\left(1-\frac{d}{2}\right)+\frac{d}{2} N^{2}
$$

then by the definition of $d$,

$$
\partial_{\eta \eta}^{2} f^{d, h}\left(\xi, 0, u_{1}(\xi, 0), u_{2}(\xi, 0)\right)=-\frac{\pi^{2}}{32 l^{2}(\Gamma)}<0 .
$$

Moreover we easily obtain that

$$
\begin{gathered}
\partial_{t t}^{2} f^{d, h}\left(\xi, 0, u_{1}(\xi, 0), u_{2}(\xi, 0)\right)=\partial_{s s}^{2} f^{d, h}\left(\xi, 0, u_{1}(\xi, 0), u_{2}(\xi, 0)\right)=-2 h\left(1-\frac{d}{2}\right), \\
\partial_{s \eta}^{2} f^{d, h}\left(\xi, 0, u_{1}(\xi, 0), u_{2}(\xi, 0)\right)=-2\left(1-\frac{d}{2}\right) \partial_{\eta \eta}^{2} u_{1}(\xi, 0) \\
\partial_{t \eta}^{2} f^{d, h}\left(\xi, 0, u_{1}(\xi, 0), u_{2}(\xi, 0)\right)=2\left(1-\frac{d}{2}\right) \partial_{\eta \eta}^{2} u_{2}(\xi, 0) \\
\partial_{t s}^{2} f^{d, h}\left(\xi, 0, u_{1}(\xi, 0), u_{2}(\xi, 0)\right)=0 .
\end{gathered}
$$

By the expressions, it follows that

$$
\begin{gathered}
\operatorname{det}\left(\begin{array}{cc}
\partial_{\eta \eta}^{2} f^{d, h} & \partial_{s \eta}^{2} f^{d, h} \\
\partial_{s \eta}^{2} f^{d, h} & \partial_{s s}^{2} f^{d, h}
\end{array}\right)\left(\xi, 0, u_{1}(\xi, 0), u_{2}(\xi, 0)\right) \\
=h(2-d) \frac{\pi^{2}}{32 l^{2}(\Gamma)}-(2-d)^{2}\left[\partial_{\eta \eta}^{2} u_{1}(\xi, 0)\right]^{2},
\end{gathered}
$$

and that the determinant of the hessian matrix of $f^{d, h}$ at $\left(\xi, 0, u_{1}(\xi, 0), u_{2}(\xi, 0)\right)$ is given by

$$
\begin{aligned}
\operatorname{det} & \nabla_{\eta s t}^{2} f^{d, h}\left(\xi, 0, u_{1}(\xi, 0), u_{2}(\xi, 0)\right) \\
& =-h^{2}(2-d)^{2} \frac{\pi^{2}}{32 l^{2}(\Gamma)}+h(2-d)^{3}\left[\left(\partial_{\eta \eta}^{2} u_{1}(\xi, 0)\right)^{2}+\left(\partial_{\eta \eta}^{2} u_{2}(\xi, 0)\right)^{2}\right] .
\end{aligned}
$$

By the definition of $d$, if $h$ satisfies

$$
h>\frac{32}{\pi^{2}}(2-d) l^{2}(\Gamma) \sum_{i=1}^{2}\left\|\partial_{\eta \eta}^{2} u_{i}\right\|_{L^{\infty}(\Gamma)}^{2},
$$

then for every $(\xi, 0) \in V$ we have

$$
\operatorname{det}\left(\begin{array}{cc}
\partial_{\eta \eta}^{2} f^{d, h} & \partial_{s \eta}^{2} f^{d, h} \\
\partial_{s \eta}^{2} f^{d, h} & \partial_{s s}^{2} f^{d, h}
\end{array}\right)\left(\xi, 0, u_{1}(\xi, 0), u_{2}(\xi, 0)\right)>0
$$


and

$$
\operatorname{det} \nabla_{\eta s t}^{2} f^{d, h}\left(\xi, 0, u_{1}(\xi, 0), u_{2}(\xi, 0)\right)<0 .
$$

By (3.43), (3.45), and (3.46), we can conclude that the hessian matrix of $f^{d, h}$ at $\left(\xi, 0, u_{1}(\xi, 0), u_{2}(\xi, 0)\right)$ is negative definite: both (3.42) and Claim 4 are proved.

CLAIM 5. - For every $r>0$ and $h>0$, there exists $\tilde{\varepsilon}>0$ with the property that, if $\varepsilon \in(0, \tilde{\varepsilon})$, one can find $\delta \in(0, \varepsilon)$ so that

$$
\begin{aligned}
& I\left(\xi, \eta, u_{2}(\xi, \eta), t\right) \in B(r)+b_{2}^{h}(\xi, \eta, t), \\
& I\left(\xi, \eta, s, u_{1}(\xi, \eta)\right) \in B(r)+b_{1}^{h}(\xi, \eta, s),
\end{aligned}
$$

provided $V$ is small enough, for every $\left|t-u_{2}(\xi, \eta)\right| \leqslant \delta,\left|s-u_{1}(\xi, \eta)\right| \leqslant \delta$.

Proof of the Claim. - By the definition of $\phi$ in $A_{6}$, we obtain that

$$
\begin{gathered}
I^{\xi}\left(\xi, \eta, u_{2}(\xi, \eta), t\right)=2\left(t-u_{2}(\xi, \eta)\right) \partial_{\xi} u_{2}(\xi, \eta), \\
I^{\eta}\left(\xi, \eta, u_{2}(\xi, \eta), t\right)=2\left(t-u_{2}(\xi, \eta)\right) \partial_{\eta} u_{2}(\xi, \eta)-M(\varepsilon-M \eta)^{-1}\left(t-u_{2}(\xi, \eta)\right)^{2} .
\end{gathered}
$$

To get the claim, we need to prove that

$$
\left(2\left(t-u_{2}\right) \partial_{\xi} u_{2}\right)^{2}+\left(-M(\varepsilon-M \eta)^{-1}\left(t-u_{2}\right)^{2}+h\left(t-u_{2}\right)^{2}+r\right)^{2}<r^{2},
$$

which is equivalent to

$$
\begin{gathered}
\left(2\left(t-u_{2}\right) \partial_{\xi} u_{2}\right)^{2}+\left(-M(\varepsilon-M \eta)^{-1}+h\right)^{2}\left(t-u_{2}\right)^{4} \\
+2 r\left(-M(\varepsilon-M \eta)^{-1}+h\right)\left(t-u_{2}\right)^{2}<0 .
\end{gathered}
$$

The conclusion follows by remarking that, if $V$ is small enough, the left-handside is less than

$$
\left(4\left(\partial_{\xi} u_{2}\right)^{2}+2 h r-\frac{2 M r}{3 \varepsilon}\right) \delta^{2}+o\left(\delta^{2}\right),
$$

which is negative if $\varepsilon$ is sufficiently small. The proof for $u_{1}$ is completely analogous.

Let us conclude the proof of the step. By Claim 4, we can find $h>0$ such that (3.40) is satisfied for $\varepsilon \in(0, \bar{\varepsilon})$. If we choose $r$ such that $2 r<d / 4$, by Claim 5 there exists $\tilde{\varepsilon}>0$ such that for every $\varepsilon \in(0, \tilde{\varepsilon})$ there is $\delta \in(0, \varepsilon)$ so that

$$
I\left(\xi, \eta, s, u_{1}(\xi, \eta)\right)+I\left(\xi, \eta, u_{2}(\xi, \eta), t\right) \in B(2 r)+b_{1}^{h}(\xi, \eta, s)+b_{2}^{h}(\xi, \eta, t)
$$

for every $\left|s-u_{1}(\xi, \eta)\right|<\delta,\left|t-u_{2}(\xi, \eta)\right|<\delta$, and $(\xi, \eta) \in V$. If we take $\varepsilon \leqslant \min \{\tilde{\varepsilon}, \bar{\varepsilon}\}$, then by Claim 4 we have that the set

$$
B(2 r)+\left(\rho_{\varepsilon}(\xi, \eta) \sin \tilde{\theta}(\eta), \rho_{\varepsilon}(\xi, \eta) \cos \tilde{\theta}(\eta)\right)+b_{1}^{h}(\xi, \eta, s)+b_{2}^{h}(\xi, \eta, t)
$$

is contained in the ball centred at $(0,0)$ with radius $\gamma(\xi, \eta)$. Some easy geometric considerations show that the relation between $\theta_{\varepsilon}$ and $\tilde{\theta}$ (see (3.38)) implies that also 
the set

$$
B(2 r)+\left(\rho_{\varepsilon}(\xi, \eta) \sin \theta_{\varepsilon}(\eta), \rho_{\varepsilon}(\xi, \eta) \cos \theta_{\varepsilon}(\eta)\right)+b_{1}^{h}(\xi, \eta, s)+b_{2}^{h}(\xi, \eta, t)
$$

is contained in the ball centred at $(0,0)$ with radius $\gamma(\xi, \eta)$, if the condition

$$
\left|b_{1}^{h}(\xi, \eta, s)+b_{2}^{h}(\xi, \eta, t)\right|<2 r
$$

holds (to make this true, take $\delta$ and $V$ smaller if needed). Since

$$
I(\xi, \eta, s, t)=I\left(\xi, \eta, s, u_{1}(\xi, \eta)\right)+I\left(\xi, \eta, u_{1}(\xi, \eta), u_{2}(\xi, \eta)\right)+I\left(\xi, \eta, u_{2}(\xi, \eta), t\right)
$$

by (3.47), (3.18), and (3.19), it follows that $I(\xi, \eta, s, t)$ belongs to the set (3.48), and then to the ball centred at $(0,0)$ with radius $\gamma(\xi, \eta)$ for every $\left|s-u_{1}(\xi, \eta)\right|<\delta$, $\left|t-u_{2}(\xi, \eta)\right|<\delta$, and $(\xi, \eta) \in V$. This concludes the proof of Step 1 .

Step 2. If $\varepsilon$ is sufficiently small and $\delta \in(0, \varepsilon)$, condition (d) holds for $\left|s-u_{1}(\xi, \eta)\right| \geqslant \delta$ or $\left|t-u_{2}(\xi, \eta)\right| \geqslant \delta$, and $(\xi, \eta) \in V$, provided $V$ is small enough.

Let us fix $\delta \in(0, \varepsilon)$ and set

$m_{1}(\xi, \eta):=\max \left\{|I(\xi, \eta, s, t)|: u_{1}(\xi, \eta)-\varepsilon \leqslant s \leqslant t \leqslant u_{2}(\xi, \eta)+\varepsilon,\left|t-u_{2}(\xi, \eta)\right| \geqslant \delta\right\}$.

It is easy to see that the function $m_{1}$ is continuous. Let us prove that $m_{1}(\xi, 0)<$ $\gamma(\xi, 0)=1$.

Fixed $(\xi, 0) \in V, u_{1}(\xi, 0)-\varepsilon \leqslant s \leqslant t \leqslant u_{2}(\xi, 0)+\varepsilon$, with $\left|t-u_{2}(\xi, 0)\right| \geqslant \delta$, we can write

$$
I(\xi, 0, s, t)=I\left(\xi, 0, s, u_{1}(\xi, 0)\right)+I\left(\xi, 0, u_{1}(\xi, 0), u_{2}(\xi, 0)\right)+I\left(\xi, 0, u_{2}(\xi, 0), t\right) .
$$

CLAIM 6. - For every $r>0$ there exists $\varepsilon>0$ such that

$$
I\left(\xi, 0, u_{2}(\xi, 0), t\right) \in B(r), \quad I\left(\xi, 0, s, u_{1}(\xi, 0)\right) \in B(r)
$$

for $0<\left|s-u_{1}(\xi, 0)\right| \leqslant \varepsilon, 0<\left|t-u_{2}(\xi, 0)\right| \leqslant \varepsilon$, and $(\xi, 0) \in V$.

Proof of the Claim. - See the similar proof of Claim 5 above.

By (3.49), (3.16), (3.17), and Claim 6, it follows that

$$
I(\xi, 0, s, t) \in(0,1)+\overline{B(r)}+B(r)=(0,1)+B(2 r)
$$

for $0<\left|s-u_{1}(\xi, 0)\right| \leqslant \varepsilon, \delta \leqslant\left|t-u_{2}(\xi, 0)\right| \leqslant \varepsilon$. If $r<1 / 4$, the set $(0,1)+B(2 r)$ is contained in the open ball centred at $(0,0)$ with radius 1 .

It remains to study the case $\left|s-u_{1}\right| \geqslant \varepsilon$ and the case $\left|t-u_{2}\right| \geqslant \varepsilon$. Let us consider the latter; the former would be completely analogous. We can write

$$
\begin{aligned}
I\left(\xi, 0, s, u_{1}(\xi, 0)\right)= & I\left(\xi, 0, s \wedge\left(u_{1}(\xi, 0)+\varepsilon\right), u_{1}(\xi, 0)\right) \\
& +I\left(\xi, 0, s \vee\left(u_{1}(\xi, 0)+\varepsilon\right), u_{1}(\xi, 0)+\varepsilon\right), \\
I\left(\xi, 0, u_{2}(\xi, 0), t\right)= & I\left(\xi, 0, u_{2}(\xi, 0), u_{2}(\xi, 0)-\varepsilon\right)+I\left(\xi, 0, u_{2}(\xi, 0)-\varepsilon, t\right) .
\end{aligned}
$$


Therefore, by (3.49)

$$
\begin{aligned}
I(\xi, 0, s, t)= & I\left(\xi, 0, u_{1}(\xi, 0), u_{2}(\xi, 0)\right)+I\left(\xi, 0, s \wedge\left(u_{1}(\xi, 0)+\varepsilon\right), u_{1}(\xi, 0)\right) \\
& +I\left(\xi, 0, u_{2}(\xi, 0), u_{2}(\xi, 0)-\varepsilon\right)+I\left(\xi, 0, s \vee\left(u_{1}(\xi, 0)+\varepsilon\right), t\right) \\
& -I\left(\xi, 0, u_{1}(\xi, 0)+\varepsilon, u_{2}(\xi, 0)-\varepsilon\right) .
\end{aligned}
$$

If $-2 \varepsilon\left(\partial_{\xi} u_{1}(\xi, 0)+\partial_{\xi} u_{2}(\xi, 0)\right) \geqslant 0$, we define

$$
C:=\left[0,-2 \varepsilon\left(\partial_{\xi} u_{1}(\xi, 0)+\partial_{\xi} u_{2}(\xi, 0)\right)\right] \times[0,1-2 \varepsilon M] ;
$$

if $-2 \varepsilon\left(\partial_{\xi} u_{1}(\xi, 0)+\partial_{\xi} u_{2}(\xi, 0)\right)<0$, we simply replace $\left[0,-2 \varepsilon\left(\partial_{\xi} u_{1}(\xi, 0)+\partial_{\xi} u_{2}(\xi, 0)\right)\right]$ by $\left[-2 \varepsilon\left(\partial_{\xi} u_{1}(\xi, 0)+\partial_{\xi} u_{2}(\xi, 0)\right), 0\right]$. From the definition of $\phi$ in $A_{3} \cup A_{4} \cup A_{5}$, it follows that

$$
I\left(\xi, 0, u_{1}(\xi, 0)+\varepsilon, u_{2}(\xi, 0)-\varepsilon\right)=\left(-2 \varepsilon\left(\partial_{\xi} u_{1}(\xi, 0)+\partial_{\xi} u_{2}(\xi, 0)\right), 1-2 \varepsilon M\right)
$$

and

$$
I(\xi, 0, s, t) \in C
$$

for $u_{1}(\xi, 0)+\varepsilon \leqslant s \leqslant t \leqslant u_{2}(\xi, 0)-\varepsilon$. Let $D:=C-\left(-2 \varepsilon\left(\partial_{\xi} u_{1}(\xi, 0)+\partial_{\xi} u_{2}(\xi, 0)\right)\right.$, $1-2 \varepsilon M)$. Since $I^{\eta}\left(\xi, 0, u_{2}(\xi, 0), u_{2}(\xi, 0)-\varepsilon\right)=-M \varepsilon$, from (3.51), (3.16), (3.17), Claim 6, (3.52), and (3.53), we obtain

$$
\begin{gathered}
I(\xi, 0, s, t) \in[(0,1)+\overline{B(r)}+B(r)] \cap\left\{(x, y) \in \mathbb{R}^{2}: y<1-\varepsilon M\right\}+D \\
=[(0,1)+B(2 r)] \cap\left\{(x, y) \in \mathbb{R}^{2}: y<1-\varepsilon M\right\}+D .
\end{gathered}
$$

If $r<1 / 4$ and if $\varepsilon$ is sufficiently small, the set $[(0,1)+B(2 r)] \cap\left\{(x, y) \in \mathbb{R}^{2}: y<\right.$ $1-\varepsilon M\}+D$ is contained in the open ball centred at $(0,0)$ with radius 1 and this means that $m_{1}(\xi, 0)<\gamma(\xi, 0)$.

Analogously we define

$m_{2}(\xi, \eta):=\max \left\{|I(\xi, \eta, s, t)|: u_{1}(\xi, \eta)-\varepsilon \leqslant s \leqslant t \leqslant u_{2}(\xi, \eta)+\varepsilon,\left|s-u_{1}(\xi, \eta)\right| \geqslant \delta\right\}$.

Arguing as in the case of $m_{1}$, we can prove that $m_{2}$ is continuous and $m_{2}(\xi, 0)<\gamma(\xi, 0)$. By continuity, if $V$ is small enough, $m_{1}(\xi, \eta)<\gamma(\xi, \eta)$ and $m_{2}(\xi, \eta)<\gamma(\xi, \eta)$, for every $(\xi, \eta) \in V$ : Step 2 is proved.

By Step 1 and Step 2, we conclude that, choosing $\varepsilon$ sufficiently small and $n=n_{\varepsilon}$ (see (3.35)), condition (d) is true for $u_{1}(\xi, \eta)-\varepsilon \leqslant s, t \leqslant u_{2}(\xi, \eta)+\varepsilon$ and in fact for every $s, t \in \mathbb{R}$, from the definition of $\phi$ in $A_{1}$ and $A_{7}$.

\section{The graph-minimality}

We start this section with a negative result: if the domain $\Omega$ is too large, the Euler conditions do not guarantee the graph-minimality introduced in Definition 1.3, as the following counterexample (suggested by Gianni Dal Maso) shows. 
Proposition 4.1. - Let $R$ be the rectangle $(1,1+4 l) \times(-l, l)$ and let

$$
u(x, y):= \begin{cases}x & \text { if } y \geqslant 0, \\ -x & \text { if } y<0 .\end{cases}
$$

Then, $u$ satisfies the Euler conditions for the Mumford-Shah functional in $R$, but it is not a local graph-minimizer in $R$ for l large enough.

Proof. - The Euler conditions are obviously satisfied by $u$ in $R$.

Let $R_{0}$ be the rectangle $(0,4) \times(-1,0)$ and let $w$ be any function in $H^{1}\left(R_{0}\right)$ such that $w(x, 0)=x$ for $x \in(0,2)$, and $w(x, y)=0$ for $(x, y) \in \partial R_{0} \backslash((0,4) \times\{0\})$.

The idea is to perturb $u$ by the rescaled function $v(x, y):=l w\left(\frac{x-1}{l}, \frac{y}{l}\right)$. We define the perturbed function

$$
\tilde{u}(x, y):= \begin{cases}x & \text { on } R_{1} \backslash T_{\varepsilon}, \\ -x+\eta(x-1) & \text { on } T_{\varepsilon}, \\ -x+\eta v(x, y) & \text { on } R_{2}\end{cases}
$$

where $\eta$ is a positive parameter and the rectangles $R_{1}, R_{2}$, and the triangle $T_{\varepsilon}$ are indicated in Fig. 1. We want to show that, if we set $c:=\int_{R_{0}}|\nabla w(x, y)|^{2} \mathrm{~d} x \mathrm{~d} y$, for every $l>c$ and for every $\varepsilon_{0}, \eta_{0}>0$ there exist $\varepsilon<\varepsilon_{0}$ and $\eta<\eta_{0}$ such that

$$
\int_{R}|\nabla u(x, y)|^{2} \mathrm{~d} x \mathrm{~d} y+\mathcal{H}^{1}\left(S_{u}\right)>\int_{R}|\nabla \tilde{u}(x, y)|^{2} \mathrm{~d} x \mathrm{~d} y+\mathcal{H}^{1}\left(S_{\tilde{u}}\right) .
$$

By definition, $\tilde{u}$ satisfies the boundary conditions. Since by the construction of $v$ the function $\tilde{u}$ is continuous on the interface between $T_{\varepsilon}$ and $R_{2}$, then

$$
\mathcal{H}^{1}\left(S_{u}\right)-\mathcal{H}^{1}\left(S_{\tilde{u}}\right)=2 l-2 \sqrt{l^{2}+\varepsilon^{2}}=-\frac{\varepsilon^{2}}{l}+\mathrm{o}\left(\varepsilon^{2}\right) .
$$

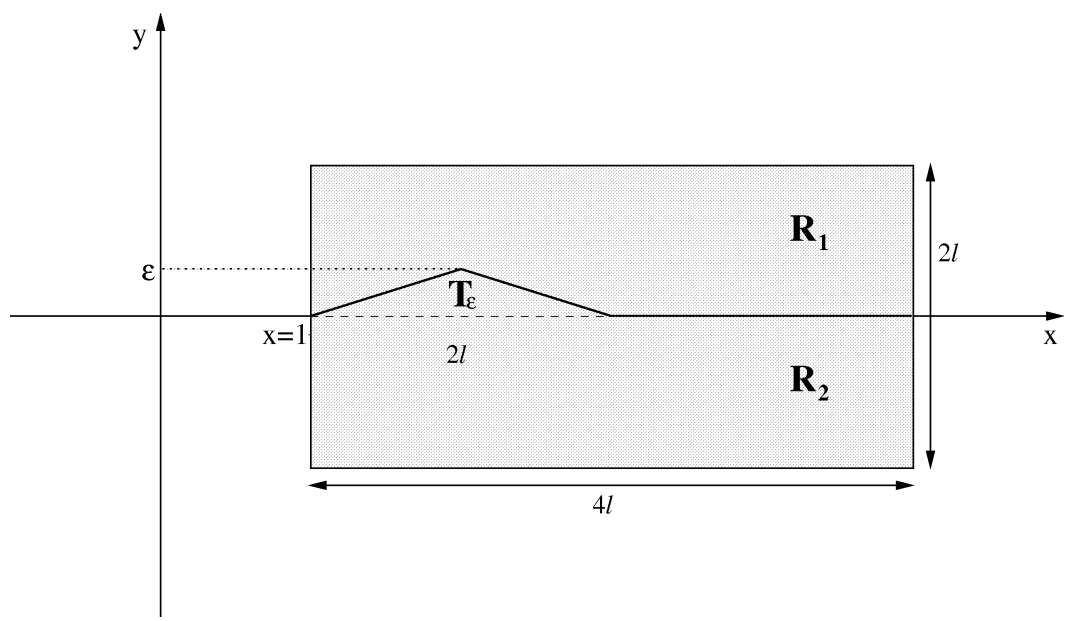

Fig. 1. The regions $R_{1}, R_{2}$ and $T_{\varepsilon}$. 
On the triangle $T_{\varepsilon}$, we obtain

$$
\int_{T_{\varepsilon}}|\nabla u(x, y)|^{2} \mathrm{~d} x \mathrm{~d} y-\int_{T_{\varepsilon}}|\nabla \tilde{u}(x, y)|^{2} \mathrm{~d} x \mathrm{~d} y=2 l \varepsilon \eta-l \varepsilon \eta^{2} .
$$

Finally, since we have that $|\nabla \tilde{u}|^{2}=1+\eta^{2}|\nabla v|^{2}-2 \eta \partial_{x} v$ in $R_{2}$, taking into account the boundary conditions of $v$, we get

$$
\begin{aligned}
\int_{R_{2}}|\nabla u(x, y)|^{2} \mathrm{~d} x \mathrm{~d} y-\int_{R_{2}}|\nabla \tilde{u}(x, y)|^{2} \mathrm{~d} x \mathrm{~d} y & =-\eta^{2} \int_{R_{2}}|\nabla v(x, y)|^{2} \mathrm{~d} x \mathrm{~d} y \\
& =-l^{2} \eta^{2} \int_{R_{0}}|\nabla w(x, y)|^{2} \mathrm{~d} x \mathrm{~d} y .
\end{aligned}
$$

In order to conclude, by (4.1), (4.2), and (4.3), we have to show that for $l$ large we can choose $\varepsilon$ and $\eta$ arbitrarily close to 0 such that

$$
-\frac{\varepsilon^{2}}{l}-c l^{2} \eta^{2}+2 l \varepsilon \eta-l \varepsilon \eta^{2}+o\left(\varepsilon^{2}\right)>0 .
$$

If we choose $\eta=\varepsilon /(c l)$, then the equality above reduces to

$$
-\frac{\varepsilon^{2}}{l}+\frac{\varepsilon^{2}}{c}+o\left(\varepsilon^{2}\right)>0
$$

which is true if $l>c$.

\subsection{Proof of Theorem 1.5}

From the definition of $d$ and $N$ (see (3.39) and Claim 3 in the proof of Theorem 1.2) it follows that there is an absolute constant $\tilde{c}>0$ (independent of $\Omega_{0}, \Omega, \Gamma$, and $u$ ) such that

$$
\tilde{c}\left(1+l^{2}(\Gamma) k^{2}(\Gamma)\right)>\frac{16}{d} .
$$

The absolute constant $c$, which appears in (1.3), is defined by

$$
c:=\max \left\{\tilde{c}, \frac{64}{\pi^{2}}\right\} .
$$

Actually, to avoid problems of boundary regularity, we shall work not exactly in $\Omega$, but in a little bit larger set. Let $\Omega^{\prime}$ be a $\Gamma$-admissible set such that $\Omega \Subset \Omega^{\prime} \Subset \Omega_{0}$, and

$$
\frac{\min _{i=1,2} K\left(\Gamma \cap \Omega^{\prime}, \Omega_{i}^{\prime}\right)}{1+l^{2}\left(\Gamma \cap \Omega^{\prime}\right)+l^{2}\left(\Gamma \cap \Omega^{\prime}\right) k^{2}\left(\Gamma \cap \Omega^{\prime}\right)}>c \sum_{i=1}^{2}\left\|\partial_{\tau} u_{i}\right\|_{C^{1}\left(\Gamma \cap \Omega^{\prime}\right)}^{2},
$$

where $\Omega_{i}^{\prime}$ denote the connected components of $\Omega^{\prime} \backslash \Gamma$. This is possible by (1.3) and by the continuity properties of $K$. 
The idea of the proof is to construct first a calibration $\varphi$ in a cylinder with base an open neighbourhood of $\Gamma \cap \Omega^{\prime}$, and then to extend $\varphi$ in a tubular neighbourhood of graph $u$.

\section{Construction of the calibration around $\Gamma$}

We essentially recycle the construction of Theorem 1.2, but we need to slightly modify the definition around the graph of $u$, in order to exploit condition (1.3) and get the extendibility.

To define the calibration $\varphi(x, y, z)$ we use the same notation and the coordinate system $(\xi, \eta)$ on $U$ (open neighbourhood of $\Gamma \cap \Omega^{\prime}$ ) introduced in the proof of Theorem 1.2. The vector field will be written as

$$
\varphi(x, y, z)=\frac{1}{\gamma^{2}(\xi(x, y), \eta(x, y))} \phi(\xi(x, y), \eta(x, y), z),
$$

where $\phi$ can be represented by

$$
\phi(\xi, \eta, z)=\phi^{\xi}(\xi, \eta, z) \tau_{\xi}+\phi^{\eta}(\xi, \eta, z) \tau_{\eta}+\phi^{z}(\xi, \eta, z) e_{z} .
$$

Given suitable parameters $\varepsilon>0$ and $\lambda>0$, we consider the following subsets of $V \times \mathbb{R}$

$$
\begin{aligned}
& A_{1}:=\left\{(\xi, \eta, z) \in V \times \mathbb{R}: u_{1}(\xi, \eta)-\varepsilon v_{1}(\xi, \eta)<z<u_{1}(\xi, \eta)+\varepsilon v_{1}(\xi, \eta)\right\}, \\
& A_{2}:=\left\{(\xi, \eta, z) \in V \times \mathbb{R}: u_{1}(\xi, \eta)+\varepsilon v_{1}(\xi, \eta)<z<u_{1}(\xi, \eta)+2 \varepsilon\right\}, \\
& A_{3}:=\left\{(\xi, \eta, z) \in V \times \mathbb{R}: u_{1}(\xi, \eta)+2 \varepsilon<z<\beta_{1}(\xi, \eta)\right\}, \\
& A_{4}:=\left\{(\xi, \eta, z) \in V \times \mathbb{R}: \beta_{1}(\xi, \eta)<z<\beta_{2}(\xi, \eta)+1 / \lambda\right\}, \\
& A_{5}:=\left\{(\xi, \eta, z) \in V \times \mathbb{R}: \beta_{2}(\xi, \eta)+1 / \lambda<z<u_{2}(\xi, \eta)-2 \varepsilon\right\}, \\
& A_{6}:=\left\{(\xi, \eta, z) \in V \times \mathbb{R}: u_{2}(\xi, \eta)-2 \varepsilon<z<u_{2}(\xi, \eta)-\varepsilon v_{2}(\xi, \eta)\right\}, \\
& A_{7}:=\left\{(\xi, \eta, z) \in V \times \mathbb{R}: u_{2}(\xi, \eta)-\varepsilon v_{2}(\xi, \eta)<z<u_{2}(\xi, \eta)+\varepsilon v_{2}(\xi, \eta)\right\},
\end{aligned}
$$

where the functions $v_{i}$ are defined as

$$
v_{1}(\xi, \eta):=1+M \eta, \quad v_{2}(\xi, \eta):=1-M \eta
$$

with $M$ positive parameter such that

$$
\begin{aligned}
c(1 & \left.+l^{2}\left(\Gamma \cap \Omega^{\prime}\right)+l^{2}\left(\Gamma \cap \Omega^{\prime}\right) k^{2}\left(\Gamma \cap \Omega^{\prime}\right)\right) \sum_{j=1}^{2}\left\|\partial_{\tau} u_{j}\right\|_{C^{1}\left(\Gamma \cap \Omega^{\prime}\right)}^{2} \\
& <M<\min _{j=1,2} K\left(\Gamma \cap \Omega^{\prime}, \Omega_{i}^{\prime}\right),
\end{aligned}
$$

while $\beta_{1}$ and $\beta_{2}$ are the solutions of the Cauchy problems (3.13). Since we suppose $u_{2}>0$ on $V$, if $\varepsilon$ is small enough, while $\lambda$ is sufficiently large, then the sets $A_{1}, \ldots, A_{7}$ are nonempty and disjoint, provided $V$ is sufficiently small.

The vector $\phi(\xi, \eta, z)$ introduced in (4.6) will be written as

$$
\phi(\xi, \eta, z)=\left(\phi^{\xi \eta}(\xi, \eta, z), \phi^{z}(\xi, \eta, z)\right),
$$


where $\phi^{\xi \eta}$ is the two-dimensional vector given by the pair $\left(\phi^{\xi}, \phi^{\eta}\right)$. We define $\phi(\xi, \eta, z)$ as follows:

$$
\begin{cases}\left(2 \nabla u_{1}-2 \frac{u_{1}-z}{v_{1}} \nabla v_{1},\left|\nabla u_{1}-\frac{u_{1}-z}{v_{1}} \nabla v_{1}\right|^{2}\right) & \text { in } A_{1}, \\ \left(2 \nabla\left(u_{1}+\varepsilon v_{1}\right)-2 \frac{u_{1}+\varepsilon v_{1}-z}{\tilde{v}_{1}} \nabla \tilde{v}_{1},\left|\nabla\left(u_{1}+\varepsilon v_{1}\right)-\frac{u_{1}+\varepsilon v_{1}-z}{\tilde{v}_{1}} \nabla \tilde{v}_{1}\right|^{2}\right) & \text { in } A_{2}, \\ \left(0, \omega_{1}(\xi, \eta)\right) & \text { in } A_{3}, \\ (\lambda \sigma(\xi, \eta) \nabla w, \mu) & \text { in } A_{4}, \\ \left(0, \omega_{2}(\xi, \eta)\right) & \text { in } A_{5}, \\ \left(2 \nabla\left(u_{2}-\varepsilon v_{2}\right)-2 \frac{u_{2}-\varepsilon v_{2}-z}{\tilde{v}_{2}} \nabla \tilde{v}_{2},\left|\nabla\left(u_{2}-\varepsilon v_{2}\right)-\frac{u_{2}-\varepsilon v_{2}-z}{\tilde{v}_{2}} \nabla \tilde{v}_{2}\right|^{2}\right) & \text { in } A_{6}, \\ \left(2 \nabla u_{2}-2 \frac{u_{2}-z}{v_{2}} \nabla v_{2},\left|\nabla u_{2}-\frac{u_{2}-z}{v_{2}} \nabla v_{2}\right|^{2}\right) & \text { in } A_{7},\end{cases}
$$

where $\nabla$ denotes the gradient with respect to the variables $(\xi, \eta)$, the functions $\tilde{v}_{i}$ are defined by

$$
\tilde{v}_{1}(\xi, \eta):=2 \varepsilon+M^{\prime} \eta, \quad \tilde{v}_{2}(\xi, \eta):=2 \varepsilon-M^{\prime} \eta
$$

while

$$
\omega_{i}(\xi, \eta):=\varepsilon^{2}\left(M+M^{\prime} \frac{v_{i}(\xi, \eta)}{\tilde{v}_{i}(\xi, \eta)}\right)^{2}-\left(\partial_{\xi} u_{i}(\xi, \eta)\right)^{2}-\left(\partial_{\eta} u_{i}(\xi, \eta)\right)^{2}
$$

for $i=1,2$, and for every $(\xi, \eta) \in V$; we take the constant $\mu$ sufficiently large in order to get the required inequality between the horizontal and the vertical components of the field (see condition (b) of Section 2), and $M^{\prime}$ so large that $\omega_{i}$ is positive in $V$, provided $V$ is small enough. We define $w$ as the solution of the Cauchy problem

$$
\left\{\begin{array}{l}
\Delta w=0, \\
w(\xi, 0)=-\frac{4 \varepsilon}{1-\varepsilon M^{\prime}-6 \varepsilon^{2} M} \int_{0}^{\xi} n(s)\left(\partial_{\xi} u_{1}(s, 0)+\partial_{\xi} u_{2}(s, 0)\right) \mathrm{d} s \\
\partial_{\eta} w(\xi, 0)=n(\xi),
\end{array}\right.
$$

where $n$ is a positive analytic function that must be chosen in a suitable way. We define

$$
\sigma(\xi, \eta):=\frac{1}{n(q(\xi, \eta))}\left(1-\varepsilon M^{\prime}-6 \varepsilon^{2} M\right)
$$

where the function $q$ is constructed in the same way as in (3.12).

Let us prove that for a suitable choice of the involved parameters the vector field is a calibration in a suitable neighbourhood $U$ of $\Gamma \cap \Omega^{\prime}$, which is equivalent to prove that $\phi$ satisfies (a), (b), (c), (d), and (e) in Section 2. The proof of conditions (a), (b), (c), and (e) is the same of Theorem 1.2. The proof of (d) is split again in two steps.

Step 1. For a suitable choice of $\varepsilon$ and of the function $n$ (see (4.8)) there exists $\delta>0$ such that condition (d) holds for $\left|s-u_{1}(\xi, \eta)\right|<\delta,\left|t-u_{2}(\xi, \eta)\right|<\delta$, and $(\xi, \eta) \in V$, provided $V$ is small enough. 
We essentially repeat the proof given in Theorem 1.2: Claims 1, 2, 3, and 4 are still valid with the same proof (up to the obvious changes due to the different definition of $\phi)$. Claim 5 must be modified as follows.

CLAIM 5. - For $h=\frac{64}{\pi^{2}} l^{2}(\Gamma) \sum_{i=1}^{2}\left\|\partial_{\xi} u_{i}\right\|_{C^{1}\left(\Gamma \cap \Omega^{\prime}\right)}^{2}$, there exist $r \in(0, d / 8)$ and $\tilde{\delta}>0$ such that for every $\delta \in(0, \tilde{\delta})$

$$
\begin{aligned}
& I\left(\xi, \eta, u_{2}(\xi, \eta), t\right) \in B(r)+b_{2}^{h}(\xi, \eta, t), \\
& I\left(\xi, \eta, s, u_{1}(\xi, \eta)\right) \in B(r)+b_{1}^{h}(\xi, \eta, s),
\end{aligned}
$$

provided $V$ is small enough, for every $\left|t-u_{2}(\xi, \eta)\right| \leqslant \delta,\left|s-u_{1}(\xi, \eta)\right| \leqslant \delta$.

Proof of the Claim. - Using the definition of $\phi$ in $A_{7}$, the claim is equivalent to prove

$$
\begin{aligned}
& \left(2\left(t-u_{2}\right) \partial_{\xi} u_{2}\right)^{2}+\left(-M(1-M \eta)^{-1}+h\right)^{2}\left(t-u_{2}\right)^{4} \\
& \quad+2 r\left(-M(1-M \eta)^{-1}+h\right)\left(t-u_{2}\right)^{2}<0 ;
\end{aligned}
$$

note that for $a_{1} \in(0,1)$ the left-handside is less than

$$
\left(4 \sum_{i=1}^{2}\left\|\partial_{\xi} u_{i}\right\|_{C^{1}\left(\Gamma \cap \Omega^{\prime}\right)}^{2}+2 h r-\frac{2 r}{1+a_{1}} M\right) \delta^{2}+\mathrm{o}\left(\delta^{2}\right),
$$

provided $V$ is small enough. To obtain the claim, it is sufficient to prove that

$$
\frac{2}{r} \sum_{i=1}^{2}\left\|\partial_{\xi} u_{i}\right\|_{C^{1}\left(\Gamma \cap \Omega^{\prime}\right)}^{2}<\frac{1}{1+a_{1}} M-h .
$$

Since by (4.7), (4.4), and (4.5) we can write

$$
M=\left(\frac{16+a_{2}}{d}+\frac{64}{\pi^{2}} l^{2}\left(\Gamma \cap \Omega^{\prime}\right)\right) \sum_{i=1}^{2}\left\|\partial_{\xi} u_{i}\right\|_{C^{1}\left(\Gamma \cap \Omega^{\prime}\right)}^{2},
$$

with $a_{2}>0$, the inequality (4.9) is equivalent to

$$
\frac{2}{r}<\left(\frac{1}{1+a_{1}}-1\right) \frac{64}{\pi^{2}} l^{2}\left(\Gamma \cap \Omega^{\prime}\right)+\frac{16+a_{2}}{d} \frac{1}{1+a_{1}},
$$

which is true if $a_{1}$ is sufficiently small and $r$ is sufficiently close to $d / 8$. The proof for $u_{1}$ is completely analogous.

To conclude the proof of the step, let $r$ and $h$ be as in Claim 5. If we choose $\varepsilon<\bar{\varepsilon}$ and $\delta \leqslant \min \{\tilde{\delta}, \varepsilon\}$, by Claim 5 we have that

$$
I\left(\xi, \eta, s, u_{1}(\xi, \eta)\right)+I\left(\xi, \eta, u_{2}(\xi, \eta), t\right) \in B(2 r)+b_{1}^{h}(\xi, \eta, s)+b_{2}^{h}(\xi, \eta, t)
$$

for every $\left|s-u_{1}(\xi, \eta)\right|<\delta,\left|t-u_{2}(\xi, \eta)\right|<\delta$, and $(\xi, \eta) \in V$; since $h$ satisfies (3.44) and $2 r<d / 4$, we can apply Claim 4 to deduce that the set

$$
B(2 r)+\left(\rho_{\varepsilon}(\xi, \eta) \sin \tilde{\theta}(\eta), \rho_{\varepsilon}(\xi, \eta) \cos \tilde{\theta}(\eta)\right)+b_{1}^{h}(\xi, \eta, s)+b_{2}^{h}(\xi, \eta, t)
$$


is contained in the ball centred at $(0,0)$ with radius $\gamma(\xi, \eta)$. Some easy geometric considerations show that the relation between $\theta_{\varepsilon}$ and $\tilde{\theta}$ (see (3.38)) implies that also the set

$$
B(2 r)+\left(\rho_{\varepsilon}(\xi, \eta) \sin \theta_{\varepsilon}(\eta), \rho_{\varepsilon}(\xi, \eta) \cos \theta_{\varepsilon}(\eta)\right)+b_{1}^{h}(\xi, \eta, s)+b_{2}^{h}(\xi, \eta, t)
$$

is contained in the ball centred at $(0,0)$ with radius $\gamma(\xi, \eta)$, if the condition

$$
\left|b_{1}^{h}(\xi, \eta, s)+b_{2}^{h}(\xi, \eta, t)\right|<2 r
$$

holds (to make this true, take $\delta$ and $V$ smaller if needed). Since

$$
I(\xi, \eta, s, t)=I\left(\xi, \eta, s, u_{1}(\xi, \eta)\right)+I\left(\xi, \eta, u_{1}(\xi, \eta), u_{2}(\xi, \eta)\right)+I\left(\xi, \eta, u_{2}(\xi, \eta), t\right),
$$

by (3.47), it follows that $I(\xi, \eta, s, t)$ belongs to the set (4.11), and then to the ball centred at $(0,0)$ with radius $\gamma(\xi, \eta)$ for every $\left|s-u_{1}(\xi, \eta)\right|<\delta,\left|t-u_{2}(\xi, \eta)\right|<\delta$, and $(\xi, \eta) \in V$. This concludes the proof of Step 1 .

Step 2. If $\varepsilon$ is sufficiently small and $\delta \in(0, \varepsilon)$, condition (d) holds for $\left|s-u_{1}(\xi, \eta)\right| \geqslant \delta$ or $\left|t-u_{2}(\xi, \eta)\right| \geqslant \delta$, and $(\xi, \eta) \in V$, provided $V$ is small enough.

By using condition (4.7), arguing as in the proof of Claim 5, we can prove the following claim.

Claim 6. - There exist $r<1 / 4$ and $\varepsilon>0$ such that

$$
I\left(\xi, 0, u_{2}(\xi, 0), t\right) \in B(r), \quad I\left(\xi, 0, s, u_{1}(\xi, 0)\right) \in B(r)
$$

for $0<\left|s-u_{1}(\xi, 0)\right| \leqslant \varepsilon, 0<\left|t-u_{2}(\xi, 0)\right| \leqslant \varepsilon$, and $(\xi, 0) \in V$.

We can conclude the proof of Step 2 in the same way as in Theorem 1.2, with the minor changes due to the different definition of the field.

By Step 1 and Step 2, we conclude that, choosing $\varepsilon$ sufficiently small and $n$ in a suitable way, condition (d) is true for $u_{1}(\xi, \eta)-\varepsilon \leqslant s, t \leqslant u_{2}(\xi, \eta)+\varepsilon$. So, $\varphi$ is a calibration.

\section{Construction of the calibration around the graph of $u$}

Now the matter is to extend the field in a tubular neighbourhood of the graph of $u$. From now on, we reintroduce the Cartesian coordinates.

Let $\Gamma_{i}$ be the curve $\eta=(-1)^{i} k$, where $k>0$. If $k$ is sufficiently small, for $i=1,2$ the curve $\Gamma_{i}$ connects two points of $\partial \Omega_{i}^{\prime}$, divides $\Omega_{i}^{\prime}$ (and then $\Omega$ ) in two connected components, and the normal vector $v_{i}$ to $\Gamma_{i}$ which points towards $\Gamma$ coincides with $(-1)^{i+1} \nabla \eta /|\nabla \eta|$. Set $U^{\prime}:=U \cap\left\{(x, y) \in \Omega^{\prime}:|\eta(x, y)|<k\right\}$ and $U^{\prime \prime}:=U^{\prime} \cap \Omega$. Since $\|\nabla \eta\|=1$ on $\Gamma$, by (4.7) we can suppose that

$$
\frac{M}{1-M k} \max _{i=1,2}\|\nabla \eta\|_{L^{\infty}\left(\Gamma_{i}\right)}<\min _{i=1,2} K\left(\Gamma_{i}, \Omega_{i}^{\prime} \backslash \overline{U^{\prime}}\right) .
$$


Chosen $\delta$ so small that $(\operatorname{graph} u)_{\delta} \cap\left(\left(U^{\prime \prime} \cap \Omega_{1}\right) \times \mathbb{R}\right) \subset A_{1}$ and $(\operatorname{graph} u)_{\delta} \cap\left(\left(U^{\prime \prime} \cap \Omega_{2}\right) \times\right.$ $\mathbb{R}) \subset A_{7}$, we define the vector field

$$
\hat{\varphi}(x, y, z)=\left(\hat{\varphi}^{x y}(x, y, z), \hat{\varphi}^{z}(x, y, z)\right) \in \mathbb{R}^{3},
$$

as follows:

$\left\{\begin{array}{cl}\varphi(x, y, z) & \text { in }\left\{(x, y, z):(x, y) \in U^{\prime \prime},\right. \\ & \left.u_{1}(x, y)-\delta<z<u_{2}(x, y)+\delta\right\} \\ \left(2 \nabla u-2 \frac{u-z}{\hat{u}_{1}} \nabla \hat{v}_{1},\left|\nabla u-\frac{u-z}{\hat{v}_{1}} \nabla \hat{v}_{1}\right|^{2}\right) & \text { in }(\operatorname{graph} u)_{\delta} \cap\left(\Omega_{1} \backslash U^{\prime \prime}\right) \times \mathbb{R}, \\ \left(2 \nabla u-2 \frac{-z}{\hat{v}_{2}} \nabla \hat{v}_{2},\left|\nabla u-\frac{u-z}{\hat{v}_{2}} \nabla \hat{v}_{2}\right|^{2}\right) & \text { in }(\operatorname{graph} u)_{\delta} \cap\left(\Omega_{2} \backslash U^{\prime \prime}\right) \times \mathbb{R} .\end{array}\right.$

The function $\hat{v}_{i}$ is the solution of the problem

$$
\min \left\{\int_{\Omega_{i}^{\prime} \backslash \overline{U^{\prime}}}|\nabla v|^{2} \mathrm{~d} x \mathrm{~d} y-\frac{M}{1-M k} \int_{\Gamma_{i}}|\nabla \eta| v^{2} \mathrm{~d} \mathcal{H}^{1}: v \in H^{1}\left(\Omega_{i}^{\prime} \backslash \overline{U^{\prime}}\right),\left.v\right|_{\partial\left(\Omega_{i}^{\prime} \backslash \overline{U^{\prime}} \backslash \backslash \Gamma_{i}\right.}=1\right\} .
$$

Let us show that the problem (4.13) admits a solution. If $\left\{v_{n}\right\}$ is a minimizing sequence, then

$$
\sup _{n} \int_{\Omega_{i}^{\prime} \backslash \bar{U}^{\prime}}\left|\nabla v_{n}\right|^{2} \mathrm{~d} x \mathrm{~d} y-\frac{M}{1-M k} \int_{\Gamma_{i}}|\nabla \eta| v_{n}^{2} \mathrm{~d} \mathcal{H}^{1}<+\infty .
$$

We have only to show that $\left\{v_{n}\right\}$ is bounded in $H^{1}\left(\Omega_{i}^{\prime} \backslash \overline{U^{\prime}}\right)$. If we put $\bar{v}_{n}:=v_{n}-1$, by (1.2) for every $\tau \in(0,1)$ we have

$$
\begin{aligned}
\int_{\Omega_{i}^{\prime} \backslash \overline{U^{\prime}}}\left|\nabla v_{n}\right|^{2} \mathrm{~d} x \mathrm{~d} y= & \int_{\Omega_{i}^{\prime} \backslash \overline{U^{\prime}}}\left|\nabla \bar{v}_{n}\right|^{2} \mathrm{~d} x \mathrm{~d} y \\
= & \left(\int_{\Gamma_{i}} \bar{v}_{n}^{2} \mathrm{~d} \mathcal{H}^{1}\right) \int_{\Omega_{i}^{\prime} \backslash \overline{U^{\prime}}}\left|\nabla\left(\frac{\bar{v}_{n}}{\left(\int_{\Gamma_{i}} \bar{v}_{n}^{2} \mathrm{~d} \mathcal{H}^{1}\right)^{1 / 2}}\right)\right|^{2} \mathrm{~d} x \mathrm{~d} y \\
\geqslant & \left(\int_{\Gamma_{i}}\left(v_{n}-1\right)^{2} \mathrm{~d} \mathcal{H}^{1}\right) K\left(\Gamma_{i}, \Omega_{i}^{\prime} \backslash \overline{U^{\prime}}\right) \\
\geqslant & (1-\tau) K\left(\Gamma_{i}, \Omega_{i}^{\prime} \backslash \overline{U^{\prime}}\right) \int_{\Gamma_{i}} v_{n}^{2} \mathrm{~d} \mathcal{H}^{1} \\
& +K\left(\Gamma_{i}, \Omega_{i}^{\prime} \backslash \overline{U^{\prime}}\right)\left(1-\frac{1}{\tau}\right) \mathcal{H}^{1}\left(\Gamma_{i}\right),
\end{aligned}
$$

where we used Cauchy Inequality. By (4.12), we can choose $\tau$ so small that

$$
(1-\tau) K\left(\Gamma_{i}, \Omega_{i}^{\prime} \backslash \overline{U^{\prime}}\right)>\frac{M}{1-M k}\|\nabla \eta\|_{L^{\infty}\left(\Gamma_{i}\right)},
$$


and substituting (4.15) in (4.14), we obtain

$$
\sup _{n} \int_{\Gamma_{i}} v_{n}^{2} \mathrm{~d} \mathcal{H}^{1}<+\infty
$$

Using again (4.14) and Poincaré Inequality, we conclude that $\left\{v_{n}\right\}$ is actually bounded in $H^{1}\left(\Omega_{i}^{\prime} \backslash \overline{U^{\prime}}\right)$.

The solution of (4.13) satisfies

$$
\begin{cases}\triangle \hat{v}_{i}=0 & \text { in } \Omega_{i}^{\prime} \backslash \overline{U^{\prime}}, \\ \frac{\partial \hat{v}_{i}}{\partial v}=\frac{M}{1-M k}|\nabla \eta| \hat{v}_{i} & \text { on } \Gamma_{i}, \\ \hat{v}_{i}=1 & \text { on } \partial\left(\Omega_{i}^{\prime} \backslash \overline{U^{\prime}}\right) \backslash \Gamma_{i},\end{cases}
$$

and so, in particular, belongs to $C^{\infty}\left(\overline{\Omega_{i} \backslash U^{\prime \prime}}\right)$. By a truncation argument, it is easy to see that $\hat{v}_{i} \geqslant 1$, so $\hat{\varphi}$ is well defined.

Since $\hat{\varphi}$ is a calibration in $\left\{(x, y, z):(x, y) \in U^{\prime \prime}, u_{1}(x, y)-\delta<z<u_{2}(x, y)+\delta\right\}$, it remains to prove only that the field is globally divergence free in the sense of distributions and that conditions (b), (c), (d) are verified in the regions (graph $u)_{\delta} \cap\left(\Omega_{i} \backslash\right.$ $\left.U^{\prime \prime}\right) \times \mathbb{R}$. First of all, note that by Lemma 3.1 the field $\hat{\varphi}$ is divergence free in the regions (graph $u)_{\delta} \cap\left(\Omega_{i} \backslash U^{\prime \prime}\right) \times \mathbb{R}$, since it is constructed starting from the family of harmonic functions $u(x, y)-t \hat{v}_{i}(x, y)$. To complete the proof, we need to check that the normal components of the traces of $\varphi$ and of the extension field are equal on the surface of separation, i.e.,

$$
\varphi^{x y} \cdot v_{i}=\left(2 \nabla u-2 \frac{u-z}{\hat{v}_{i}} \nabla \hat{v}_{i}\right) \cdot v_{i} \quad \text { on } \Gamma_{i},
$$

where $v_{i}=(-1)^{i+1} \nabla \eta /|\nabla \eta|$. Using the definition of $\varphi$, we obtain that

$$
\varphi^{x y} \cdot v_{i}=\left((-1)^{i+1} \partial_{\eta} u-\frac{u-z}{1-M k} M\right)|\nabla \eta|
$$

since $\nabla u \cdot v_{i}=(-1)^{i+1} \partial_{\eta} u|\nabla \eta|$, equality (4.17) is equivalent to

$$
\frac{M}{1-M k}|\nabla \eta|=\frac{1}{\hat{v}_{i}} \nabla \hat{v}_{i} \cdot v_{i}
$$

which is true by (4.16).

Conditions (b) and (c) are obviously satisfied, while condition (d) is true if we take $\delta$ satisfying

$$
\delta \leqslant \sup \left\{\left(4|\nabla u|+2 \frac{\left|\nabla \hat{v}_{i}\right|}{\hat{v}_{i}}\right)^{-1}:(x, y) \in \Omega_{i} \backslash U^{\prime \prime}, i=1,2\right\}
$$

Therefore, with this choice of $\delta$, the vector field $\hat{\varphi}$ is a calibration. 


\subsection{Some properties of $K(\Gamma, A)$}

In this subsection we investigate some qualitative properties of the quantity $K(\Gamma, A)$ and we shall compute it explicitly in a very particular case. Let us start by a very simple result.

Proposition 4.2. - Let $\Gamma$ be a simple analytic curve and $\tilde{\Gamma}$ an extension of $\Gamma$, whose endpoints do not coincide with the endpoints of $\Gamma$. If $\Gamma_{\delta}^{ \pm}$are the two connected components of $\Gamma_{\delta} \backslash \tilde{\Gamma}$ (which are well defined if $\delta$ is sufficiently small), then

$$
\lim _{\delta \rightarrow 0^{+}} K\left(\Gamma, \Gamma_{\delta}^{ \pm}\right)=+\infty .
$$

Proof. - For convenience we set

$$
W^{ \pm}(\delta):=\left\{v \in H^{1}\left(\Gamma_{\delta}^{ \pm}\right): \int_{\Gamma} v^{2} \mathrm{~d} \mathcal{H}^{1}=1, v=0 \text { on } \partial\left(\Gamma_{\delta}^{ \pm}\right) \backslash \Gamma\right\} .
$$

Suppose by contradiction that there exists a sequence $\left\{\delta_{n}\right\}$ decreasing to 0 such that $\sup _{n} K\left(\Gamma, \Gamma_{\delta_{n}}^{+}\right)=c<+\infty$; this implies the existence of a sequence $\left\{v_{n}\right\}$ such that

$$
v_{n} \in W^{+}\left(\delta_{n}\right) \quad \text { and } \quad \int_{\Gamma_{\delta_{n}}^{+}}\left|\nabla v_{n}(x, y)\right|^{2} \mathrm{~d} x \mathrm{~d} y \leqslant c
$$

for every integer $n$. From now on, we regard $v_{n}$ as a function belonging to $H^{1}\left(\Gamma_{\delta_{1}}^{+}\right)$ which vanishes on $\Gamma_{\delta_{1}}^{+} \backslash \Gamma_{\delta_{n}}^{+}$. By Poincaré Inequality it follows immediately that $\left\{v_{n}\right\}$ is bounded in $H^{1}\left(\Gamma_{\delta_{1}}^{+}\right)$, and so admits a weakly convergent subsequence $\left\{v_{n_{k}}\right\}$. Let us call $v$ the limit of the subsequence; since for every $k, v_{n_{k}}$ vanishes on $\Gamma_{\delta_{1}}^{+} \backslash \Gamma_{\delta_{n_{k}}}^{+}$, then $v$ must vanish a.e.; on the other hand, since $\int_{\Gamma} v_{n_{k}}^{2} \mathrm{~d} \mathcal{H}^{1}=1$, by the compactness of the trace operator, we have that $\int_{\Gamma} v^{2} \mathrm{~d} \mathcal{H}^{1}=1$, and this is clearly impossible.

We remark that by Theorem 1.5 and Proposition 4.2, if $U_{0}$ is a neighbourhood of $\Gamma$ and $u \in \operatorname{SBV}\left(U_{0}\right)$ satisfies the Euler conditions in $U_{0}$ with $S_{u}=\Gamma$, then there exists a neighbourhood $U$ of $\Gamma$ contained in $U_{0}$ such that $u$ is a local graph-minimizer in $U$. Actually, taking $U$ smaller if needed, by Theorem 1.2 we get also the Dirichlet minimality.

Proposition 4.3 (Characterization of $K(\Gamma, A)$ ). - Let $A$ be an open set with Lipschitz boundary and $\Gamma$ be a subset of $\partial A$ with nonempty relative interior in $\partial A$. The constant $K(\Gamma, A)$ is the first eigenvalue of the problem

$$
\begin{cases}\Delta u=0 & \text { on } A, \\ \partial u / \partial v=\lambda u & \text { on } \Gamma, \\ u=0 & \text { on } \partial A \backslash \Gamma .\end{cases}
$$

Moreover, it is the unique eigenvalue with a positive eigenfunction. 
Proof. - If $u$ is a solution of (1.2), then it is harmonic and there exists a Lagrange multiplier $\lambda$ such that

$$
2 \int_{A} \nabla u \cdot \nabla \varphi \mathrm{d} x \mathrm{~d} y=\lambda \int_{\Gamma} u \varphi \mathrm{d} \mathcal{H}^{1} \quad \forall \varphi \in C^{\infty}(A): \varphi=0 \text { on } \partial A \backslash \Gamma,
$$

which means, by Green Formula, that $\partial u / \partial v=\lambda u$ on $\Gamma$. Using (4.19), one can easily see that $K(\Gamma, A)$ is in fact the minimal eigenvalue of (4.18) and that it has a positive eigenfunction (indeed, if $u$ is a solution also $|u|$ is). Let $u$ be a positive function belonging to the eigenspace of $K(\Gamma, A)$ and $v$ another positive eigenfunction associated with the eigenvalue $\mu$; by Green Formula we have

$$
\int_{\Gamma} v \frac{\partial u}{\partial v} \mathrm{~d} \mathcal{H}^{1}-\int_{\Gamma} u \frac{\partial v}{\partial v} \mathrm{~d} \mathcal{H}^{1}=0,
$$

therefore

$$
(K(\Gamma, A)-\mu) \int_{\Gamma} u v \mathrm{~d} \mathcal{H}^{1}=0 .
$$

Since both $u$ and $v$ are positive, from the last equality it follows that $\mu=K(\Gamma, A)$.

Proposition 4.4. - If $A=(0, a) \times(0, b)$ and $\Gamma=(0, a) \times\{0\}$, then

$$
K(\Gamma, A)=\frac{\pi}{a \tanh \left(\frac{\pi b}{a}\right)} .
$$

Proof. - The function

$$
v(x, y)=\sin \left(\frac{\pi}{a} x\right) \sinh \left(\frac{\pi}{a}(b-y)\right)
$$

is positive and satisfies (4.18) with $\lambda=\pi /(a \tanh (\pi b / a))$. Then, by Proposition 4.3, this quantity coincides with $K(\Gamma, A)$.

Proposition 4.5. - Let $g:\left[0, a_{0}\right] \rightarrow[0,+\infty)$ be a Lipschitz function and denote the graph of $g$ by $\Gamma$. Given $0 \leqslant a_{1}<a_{2} \leqslant a_{0}$ and $b>0$, if we set $\Gamma\left(a_{1}, a_{2}\right):=$ graph $\left.g\right|_{\left(a_{1}, a_{2}\right)}$ and

$$
R\left(a_{1}, a_{2}, b\right):=\left\{(x, y): x \in\left(a_{1}, a_{2}\right), y \in(g(x), g(x)+b)\right\},
$$

then

$$
\lim _{\left|a_{2}-a_{1}\right| \rightarrow 0} K\left(\Gamma\left(a_{1}, a_{2}\right), R\left(a_{1}, a_{2}, b\right)\right)=+\infty \quad \text { uniformly with respect to } b .
$$

Proof. - The idea is to transform the region $R\left(a_{1}, a_{2}, b\right)$ into the rectangle $\left(0, a_{2}-\right.$ $\left.a_{1}\right) \times(0, b)$ by a suitable diffeomorphism in order to use (4.20). 
Let $\psi:\left(0, a_{2}-a_{1}\right) \times(0, b) \rightarrow R\left(a_{1}, a_{2}, b\right)$ be the map defined by $\psi(x, y)=\left(x+a_{1}\right.$, $\left.y+g\left(x+a_{1}\right)\right)$. Let $v \in H^{1}\left(R\left(a_{1}, a_{2}, b\right)\right)$ be such that $v=0$ on $\partial R\left(a_{1}, a_{2}, b\right) \backslash \Gamma\left(a_{1}, a_{2}\right)$ and

$$
\int_{\Gamma\left(a_{1}, a_{2}\right)} v^{2} \mathrm{~d} \mathcal{H}^{1}=\int_{0}^{a_{2}-a_{1}} v^{2}(\psi(x, 0)) \sqrt{1+\left(g^{\prime}(x)\right)^{2}} \mathrm{~d} x=1 .
$$

If we call $\tilde{v}(x, y):=v(\psi(x, y))$, then $\tilde{v} \in H^{1}\left(\left(0, a_{2}-a_{1}\right) \times(0, b)\right), \tilde{v}=0$ on the boundary of the rectangle except $\left(0, a_{2}-a_{1}\right) \times\{0\}$, and by (4.21) there exists $\lambda>0$ such that $\lambda^{2} \leqslant \sqrt{1+\left\|g^{\prime}\right\|_{\infty}^{2}}$ and

$$
\lambda^{2} \int_{0}^{a_{2}-a_{1}} \tilde{v}^{2}(x, 0) \mathrm{d} x=1
$$

Therefore, since $J \psi \equiv 1$,

$$
\begin{aligned}
& \int_{R\left(a_{1}, a_{2}, b\right)}|\nabla v(x, y)|^{2} \mathrm{~d} x \mathrm{~d} y \\
& =\int_{\left(0, a_{2}-a_{1}\right) \times(0, b)}|\nabla v(\psi(x, y))|^{2} \mathrm{~d} x \mathrm{~d} y \\
& \geqslant\left(1+\left\|g^{\prime}\right\|_{\infty}+\left\|g^{\prime}\right\|_{\infty}^{2}\right)^{-1} \int_{\left(0, a_{2}-a_{1}\right) \times(0, b)}|\nabla \tilde{v}(x, y)|^{2} \mathrm{~d} x \mathrm{~d} y \\
& \geqslant \lambda^{-2}\left(1+\left\|g^{\prime}\right\|_{\infty}+\left\|g^{\prime}\right\|_{\infty}^{2}\right)^{-1} K\left(\left(0, a_{2}-a_{1}\right) \times\{0\},\left(0, a_{2}-a_{1}\right) \times(0, b)\right) \\
& \geqslant\left(1+\left\|g^{\prime}\right\|_{\infty}^{2}\right)^{-3 / 2} \frac{\pi}{2\left(a_{2}-a_{1}\right) \tanh \left(\frac{\pi b}{a_{2}-a_{1}}\right)},
\end{aligned}
$$

where the last inequality follows by the estimate on $\lambda$ and by (4.20). Since $v$ is arbitrary, using the fact that $0<\tanh t \leqslant 1$ for every $t>0$, we obtain that

$$
K\left(\Gamma\left(a_{1}, a_{2}\right), R\left(a_{1}, a_{2}, b\right)\right) \geqslant\left(1+\left\|g^{\prime}\right\|_{\infty}\right)^{-3 / 2} \frac{\pi}{2\left(a_{2}-a_{1}\right)} ;
$$

so, the conclusion is clear.

We have already remarked (see Proposition 4.2) that the graph-minimality is guaranteed in small neighbourhoods of the discontinuity set $\Gamma$. As consequence of Proposition 4.5, we obtain that the graph-minimality holds also in the open sets, which are narrow along the direction parallel to $\Gamma$ and may be very large along the normal direction. This is made precise by the following corollary.

COROLlaRY 4.6. - Let $g$ be a positive function, analytic on $\left[0, a_{0}\right]$, that is $g$ admits an analytic extension, and denote the graph of $g$ by $\Gamma$. For every $M>0$ there exists $h=h(M, \Gamma)$ such that, if $\Omega$ is $\Gamma$-admissible (see Definition 1.4) and $\Omega \subset\left(a_{1}, a_{1}+h\right) \times \mathbb{R}$ with $a_{1} \in\left[0, a_{0}-h\right]$, and if $u$ is a function in $\operatorname{SBV}(\Omega)$ with 


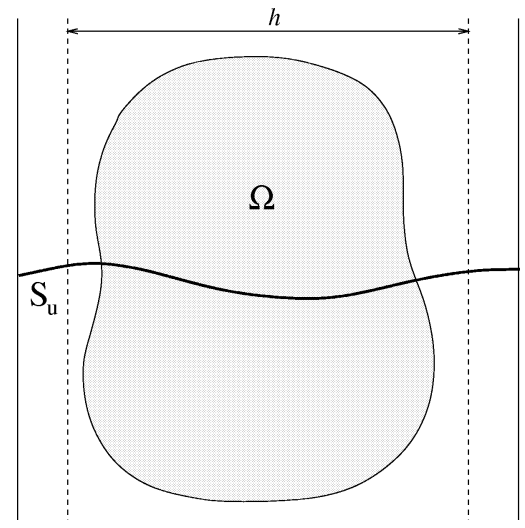

Fig. 2. If the thickness of $\Omega$ is less than $h$, then $u$ is a local graph-minimizer in $\Omega$.

$S_{u}=\Gamma \cap \Omega$, with different traces at every point of $\Gamma \cap \Omega$, satisfying the Euler conditions in $\Omega$, and $\sum_{i=1}^{2}\left\|\partial_{\tau} u_{i}\right\|_{C^{1}(\Gamma \cap \Omega)} \leqslant M$ (where $u_{i}$ is as above the restriction of $u$ to the connected component $\Omega_{i}$ of $\Omega \backslash \Gamma$ ), then $u$ is a local graph-minimizer in $\Omega$ (see Fig. 2).

Proof. - By Proposition 4.5 there exists $h>0$ such that for every $a_{1}, a_{2} \in\left[0, a_{0}\right]$ with $0<a_{2}-a_{1} \leqslant h$ and for every $b>0$,

$$
\frac{K\left(\Gamma\left(a_{1}, a_{2}\right), R\left(a_{1}, a_{2}, b\right)\right)}{1+l^{2}(\Gamma)+l^{2}(\Gamma) k^{2}(\Gamma)}>c M^{2} .
$$

If $\Omega \subset\left(a_{1}, a_{1}+h\right) \times \mathbb{R}$, then we can choose $b>0$ so large that, assuming that $\Omega_{1}$ is the upper component, $\Omega_{1} \subset R\left(a_{1}, a_{1}+h, b\right)$. Then by the monotonicity properties of $K(\Gamma, A)$, it follows that

$$
\frac{K\left(\Gamma \cap \Omega, \Omega_{1}\right)}{1+l^{2}(\Gamma)+l^{2}(\Gamma) k^{2}(\Gamma)}>c M^{2} \geqslant c \sum_{i=1}^{2}\left\|\partial_{\tau} u_{i}\right\|_{C^{1}(\Gamma \cap \Omega)}^{2}
$$

Applying the same argument to $\Omega_{2}$, the conclusion follows from Theorem 1.5.

\section{Acknowledgements}

We would like to thank Gianni Dal Maso for many helpful discussions and for having suggested to us the study of this problem.

\section{REFERENCES}

[1] Alberti G., Bouchitté G., Dal Maso G., The calibration method for the Mumford-Shah functional, Preprint SISSA, Trieste, 1998.

[2] Ambrosio L., A compactness theorem for a new class of variational problems, Boll. Un. Mat. It. 3-B (1989) 857-881.

[3] Ambrosio L., Fusco N., Pallara D., Functions of Bounded Variation and Free-Discontinuity Problems, Oxford University Press, Oxford, 2000. 
[4] Chavel I., Riemannian Geometry - A Modern Introduction, Cambridge University Press, Cambridge, 1993.

[5] Dal Maso G., Mora M.G., Morini M., Local calibrations for minimizers of the MumfordShah functional with rectilinear discontinuity set, J. Math. Pures Appl. 79 (2) (2000) 141162.

[6] Hartman P., Ordinary Differential Equations, Birkhäuser, Boston, 1982.

[7] John F., Partial Differential Equations, Springer-Verlag, New York, 1982.

[8] Mumford D., Shah J., Boundary detection by minimizing functionals, I, in: Proc. IEEE Conf. on Computer Vision and Pattern Recognition, San Francisco, 1985.

[9] Mumford D., Shah J., Optimal approximation by piecewise smooth functions and associated variational problems, Comm. Pure Appl. Math. 42 (1989) 577-685. 